\title{
CATÁLISE ASSIMÉTRICA NA CICLOPROPANAÇÃO DE OLEFINAS
}

Raquel A. C. Leão, Vítor F. Ferreira* e Sergio Pinheiro

Departamento de Química Orgânica, Instituto de Química, Universidade Federal Fluminense, Outeiro de S. João Batista, s/n, 24020-150 Niterói - RJ, Brasil

Recebido em 8/8/06; aceito em 9/11/07; publicado na web em 24/7/07

ASYMMETRIC CATALYSIS IN THE CYCLOPROPANATION OF OLEFINS.The main methodologies in the asymmetric cyclopropanation of alkenes with emphasis on asymmetric catalysis are covered. Exemples are the Simmons-Smith reaction, the use of diazoalkanes and reactions carried out by decomposition of $\alpha$-diazoesters in the presence of transition metals.

Keywords: cyclopropanation; alkenes; metalocarbenoids.

\section{INTRODUÇÃO}

Os compostos contendo o anel ciclopropano desempenham um papel importante como materiais de partida e intermediários em síntese orgânica, pois são convertidos em uma variedade de produtos úteis pela clivagem do anel ciclopropano ${ }^{1,2}$. Além disso, a unidade ciclopropano é o elemento estrutural de uma grande variedade de produtos naturais, incluindo terpenos, feromônios, ácidos graxos e aminoácidos não usuais ${ }^{3,4}$.

Nos últimos anos têm surgido revisões acerca da síntese assimétrica de produtos naturais biologicamente ativos contendo o sistema ciclopropano ${ }^{5}$. Por ex., diferentes abordagens foram descritas para as sínteses assimétricas da Antroplalona e da Norantroplona, substâncias isoladas da Anthopleura pacifica ${ }^{6}$, que exibem citotoxidade contra as células do melanoma B-16 (Figura 1). Outros exemplos incluem a Halicholactona, um inibidor da enzima 5-lipoxigenase isolada de Halichondria okadai ${ }^{7}$, as Solandelactonas C e D isoladas a partir da Solanderia secunda ${ }^{8}$, contendo atividades inibidoras da enzima farnesil transferase e a Curacina A, uma substância isolada da cianobactéria Lygnbya majuscula, que exibe atividade citotóxica para células de leucemia L1210 e para células CA46 do linfoma de Burkitt'. Dentre os compostos ciclopropânicos de origem sintética de consideráveis valores agregados podem ser mencionados a Cilastatina, que foi desenvolvida pela Merck como um inibidor seletivo da diidropeptidase renal $^{10}$, suprimindo o metabolismo do antibiótico $\beta$-lactâmico Imipenem. Outros exemplos são as arilpiperazinas I e II identificadas como potentes antagonistas de receptores dopaminérgicos com reduzidas afinidades frente a receptores adrenérgicos $\alpha_{1}{ }_{1}^{11}$.

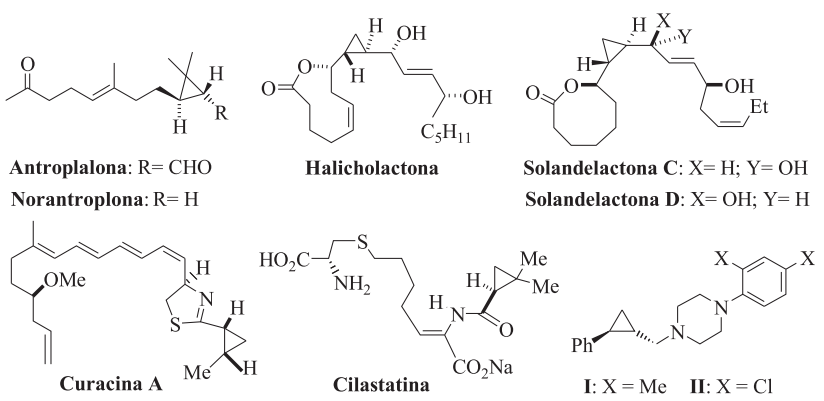

Figura 1. Alguns compostos ciclopropânicos biologicamente ativos

*e-mail: cegvito@vm.uff.br
Uma vez que compostos ciclopropânicos naturais e sintéticos, com destaque para os inseticidas piretróides, apresentam importância biológica ${ }^{12}$, muitos esforços têm objetivado o desenvolvimento de métodos diastereo- e enantiosseletivos para a construção de anéis ciclopropânicos ${ }^{5,13}$.

As principais metodologias para as sínteses de compostos ciclopropânicos quirais envolvem a reação de ciclopropanação assimétrica de olefinas, para a qual existem excelentes textos didáticos ${ }^{14}$ e revisões recentes envolvendo processos diastereosseletivos ("chiral pool") ${ }^{15}$ e enantiosseletivos ${ }^{16}$.

Existem três tipos de reação de ciclopropanação estereosseletiva com olefinas ${ }^{16}$ : reações de ciclopropanação mediada por compostos halometil-metálicos, denominada reação de Simmons-Smith (Equação 1); decomposição de diazocompostos catalisada por metais de transição (Equação 2); e reações de adição nucleofílica seguidas por fechamento de anel, onde $\mathrm{G}$ é um grupo eletroatrator e LG um grupo de saída (Equações 3 e 4). Os processos estereosseletivos representados pelas Equações 1 e 2 envolvem o emprego de catálise assimétrica.

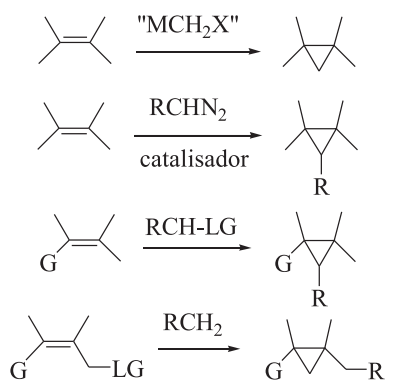

Nesta revisão serão abordadas as principais metodologias para a ciclopropanação enantiosseletiva de olefinas empregando-se catálise assimétrica.

\section{CICLOPROPANAÇÃO DE SIMMONS-SMITH}

A inserção oxidativa do zinco no $\mathrm{CH}_{2} \mathrm{I}_{2}$ produz o iodeto de iodometilzinco $\left(\mathrm{IZnCH}_{2} \mathrm{I}\right)$, que coexiste com o bis(iodometil)zinco $\left(\mathrm{Zn}\left(\mathrm{CH}_{2} \mathrm{I}_{2}\right)_{2}\right)$ no equilíbrio de Schlenk:

$\mathrm{Zn}\left(\mathrm{CH}_{2} \mathrm{I}_{2}\right)_{2}+\mathrm{ZnI}_{2} \cdot 2 \mathrm{ICH}_{2} \mathrm{ZnI}$ 
$\mathrm{O}$ reagente iodeto de iodometilzinco $\left(\mathrm{IZnCH}_{2} \mathrm{I}\right)$ faz a conversão de alcenos em ciclopropanos empregando-se processos diastereosseletivos envolvendo tanto materiais de partida como auxiliares quirais ${ }^{17}$. A reação de Simmons-Smith usando esse reagente é caracteristicamente estereoespecífica, produzindo um estado de transição do tipo "borboleta"18 (Esquema 1). Uma das maiores vantagens desta reação é a quimiossele tividade, visto que é aplicável a uma variedade de olefinas, sendo compatível com a presença de vários grupos funcionais, tais como enaminas, enol, éteres, ésteres, cetonas etc. Contudo, a ciclopropanação enantiosseletiva de olefinas tri- e tetra-substituídas geralmente não é efetiva.

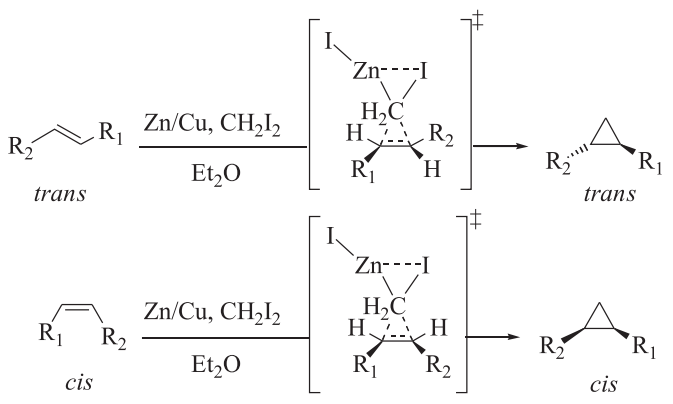

Esquema 1. Ciclopropanação estereoespecífica de Simmons-Smith

Kobayashi e colaboradores ${ }^{19}$ foram os primeiros a relatar a reação de Simmons-Smith sob condições de catálise assimétrica (Esquema 2). Esta ciclopropanação consiste na reação de álcoois alílicos com o sistema $\mathrm{Et}_{2} \mathrm{Zn} / \mathrm{CH}_{2} \mathrm{I}_{2}$ para formar ciclopropilmetanóis.

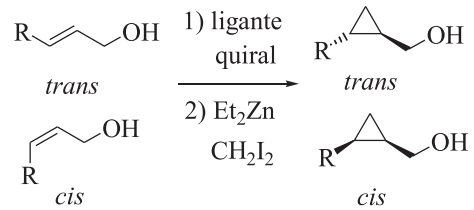

Esquema 2. Reação de Simmons-Smith: ciclopropanação de álcoois alílicos

Nesta reação, Kobayashi e colaboradores ${ }^{19}$ observaram altas enantiosseletividades pelo emprego de quantidades catalíticas da bis(sulfonamida) 1 como ligante quiral de simetria $\mathrm{C}_{2}$ (Tabela 1). Posteriormente, Denmark et al. ${ }^{20}$ estudaram profundamente essa reação e deram contribuições importantes acerca do efeito de muitas variáveis na otimização sobre o excesso enantiomérico do produto. Estes autorea mostraram que a razão e a seletividade da ciclopropanação catalítica enantiosseletiva do álcool cinamílico utilizando iodeto de iodometilzinco $\left(\mathrm{IZnCH}_{2} \mathrm{I}\right)$ e a bis(sulfonamida) 2 são fortemente dependentes da ordem de adição dos reagentes; a pré-formação independente do alcóxido de etilzinco e bis(iodometil)zinco é crucial e a reação mostrou um procedimento auto-catalítico, o qual foi atribuído à formação de $\mathrm{ZnI}_{2}$ no meio reacional. Estas condições reacionais otimizadas, usando a bis(sulfonamida) 2 como ligante quiral, permitiram a formação dos correspondentes trans-ciclopropilmetanóis em maiores enantiosseletividades.

Na reação de Simmons-Smith o heteroátomo presente no substrato atua como um grupo diretor, tanto para aumentar a velocidade da reação via um efeito de proximidade, quanto para possibilitar a formação de um estado de transição altamente ordenado, de forma a permitir um elevado estereocontrole na reação. Estas observações levaram à proposição de um modelo de estado de transição para o uso do ligante quiral $\mathbf{2}$ em que o oxigênio do álcool

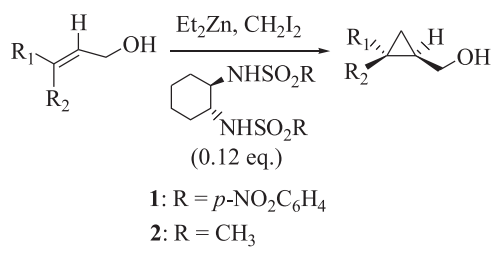

Tabela 1. Ciclopropanação de álcoois alílicos: dissulfonamidas como ligantes quirais

\begin{tabular}{lcccc}
\hline & & & \multicolumn{2}{c}{$\%$ e. e.; \% } \\
Entr. & $\mathrm{R}_{1}$ & $\mathrm{R}_{2}$ & ligante 1 & ligante 2 \\
\hline 1 & $\mathrm{Ph}$ & $\mathrm{H}$ & $76 ; 82$ & $89 ; 92^{\mathrm{a}}$ \\
2 & $\mathrm{H}$ & $\mathrm{Ph}$ & $75 ; 71$ & $81 ; 81$ \\
3 & $\mathrm{BnCH}_{2}$ & $\mathrm{H}$ & $82 ; 100$ & $89 ; 88^{\mathrm{a}}$ \\
4 & $\mathrm{H}$ & $\mathrm{BnCH}_{2}$ & $72 ; 93$ & $75 ; 93$ \\
\hline
\end{tabular}

${ }^{a} \mathrm{ZnI}_{2}$ gerado in situ

alílico coordena três átomos de zinco, os quais se encontram envolvidos no processo de transferência de metileno ${ }^{20}$ (Figura 2).

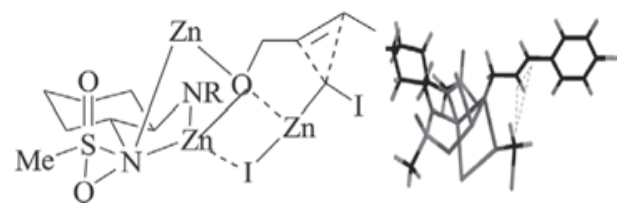

Figura 2. Estado de transição na ciclopropanação de álcoois alílicos

Charette e Brochu ${ }^{21}$ relataram um protocolo alternativo para a ciclopropanação de álcoois alílicos catalisada por ácidos de Lewis, em que o processo não catalisado é minimizado. A adição do $\mathrm{Zn}\left(\mathrm{CH}_{2} \mathrm{I}\right)_{2}$ (1 equiv.) ao álcool alílico (1 equiv.) produz um alcóxido de iodometilzinco A (Esquema 3). Essas espécies tipicamente não são bons agentes transferidores de metileno, a menos que um ácido de Lewis seja adicionado para a conversão de $\mathbf{B}$ ao alcóxido $\mathbf{C}$ coordenado ao ácido de Lewis. No ciclo catalítico esta espécie produz o alcóxido $\mathbf{D}$, regenerando o ácido de Lewis.

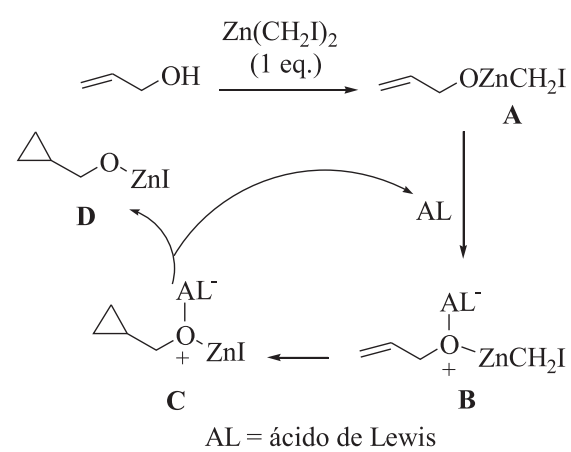

Esquema 3. Reação de Simmons-Smith: o ciclo catalítico

Resultados melhores na ciclopropanação assimétrica de Simmons-Smith de álcoois alílicos foram relatados por Charette e colaboradores ${ }^{22}$ (Equação 5). Estes autores mostraram que a pré-complexação da quantidade estequiométrica do ligante quiral 3 seguida por ciclopropanação levou ao respectivo ciclopropilcarbinol em altos rendimentos e com excelente excesso enantiomérico. Deve-se, contudo, considerar que este método apresenta o inconveniente da necessidade do uso de quantidades estequiométricas do ligante quiral 3. 


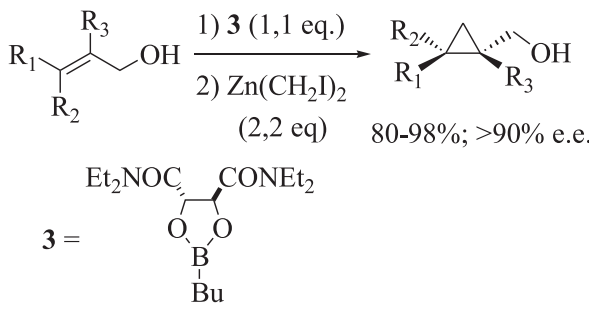

Charette et $a l .{ }^{22}$ propuseram que, neste caso, a origem da enantiosseletividade decorre da coordenação da espécie $\mathrm{Zn}\left(\mathrm{CH}_{2} \mathrm{I}\right)_{2}$, tanto pelo oxigênio da função álcool alílico quanto pela carbonila amídica, o que orienta o ataque de carbeno à ligação dupla ${ }^{22}$ (Figura 3).

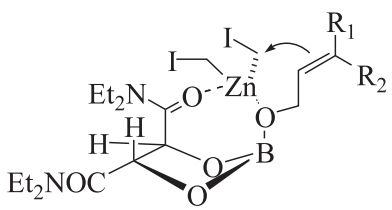

Figura 3. Origem da enantiosseletividade da ciclopropanação com o ligante $\mathbf{3}$.

Muitos outros ligantes quirais foram aplicados na reação de Simmons-Smith assimétrica a partir do álcool cinamílico ${ }^{20,23-26}$. Na Figura 4, para cada ligante, são mostrados os rendimentos químicos e as enantiosseletividades (\% e.e.) do trans-(2-fenilciclopropil)metanol formado. Embora bis(sulfonamidas), como 2 e 4, sejam os ligantes quirais mais investigados, os compostos 5-7 também se mostraram eficientes. Enquanto os ligantes 4-6 produzem o $(R, R)$ (2-fenilciclopropil)-metanol, o emprego do ligante 7 leva a formação do isômero $(S, S)$. Nestas reações os ligantes acíclicos 8-10 mostraram-se ineficientes no controle da estereosseleção.

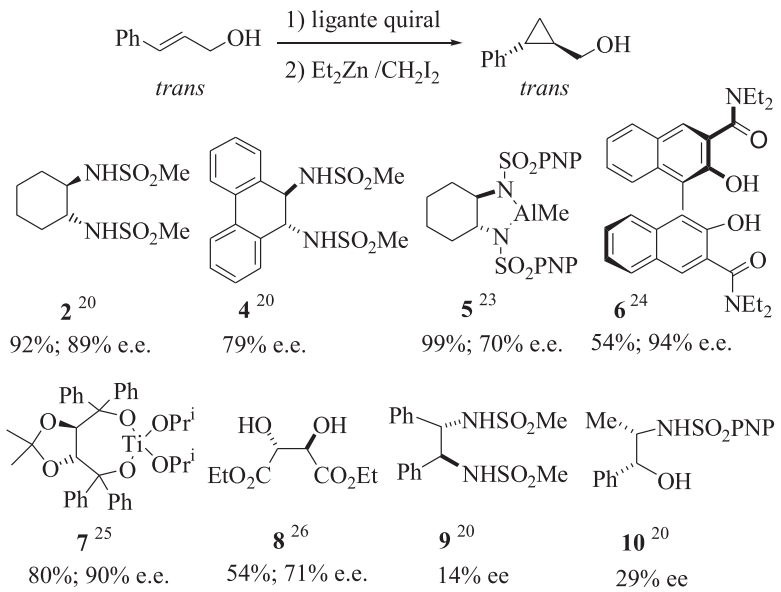

Figura 4. Principais ligantes quirais na reação de Simmons-Smith

Recentemente Shi e colaboradores ${ }^{27}$ descreveram os primeiros exemplos da ciclopropanação assimétrica de Simmons-Smith a partir de olefinas não funcionalizadas e de silil-enol-éteres, empregando o dipeptídeo 11 como ligante quiral na presença de metoxiacetato de etila como aditivo (Equações 6 e 7).

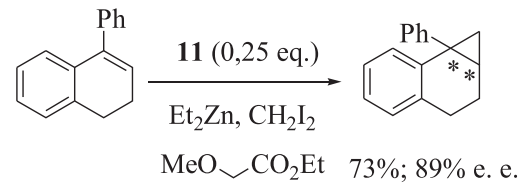

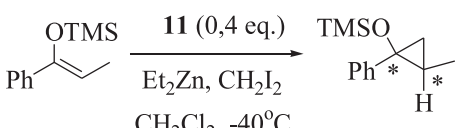

$$
\begin{aligned}
& \mathrm{CH}_{2} \mathrm{Cl}_{2},-40^{\circ} \mathrm{C} \quad 99 \% \text {; } 93 \% \text { e. e. } \\
& 11={\underset{H}{H}}_{\mathrm{O}}^{\text {Boc. }} \\
& \mathrm{R}=\mathrm{OMe}, \mathrm{Ph}
\end{aligned}
$$

\section{CICLOPROPANAÇÃO COM DIAZOALCANOS}

A ciclopropanação de olefinas por meio da decomposição de diazoalcanos na presença de catalisadores de metais de transição é uma reação bem explorada em síntese orgânica ${ }^{14,28}$ (Esquema 4). O mecanismo da extrusão de nitrogênio catalisado por metais de transição a partir de diazocompostos não é bem conhecido, mas é bem aceito que complexos metalocarbenos são formados nesse processo, embora as tentativas de identificar essas espécies nas reações catalíticas não tenham sido bem sucedidas ${ }^{14}$. Após a interação de diazoalcanos com catalisadores de metais de transição (MLn), ocorre a extrusão de $\mathrm{N}_{2}$ com a conseqüente formação de um metalocarbeno. Esta espécie é atacada pela ligação dupla da olefina, gerando o ciclopropano e regenerando o catalisador MLn.

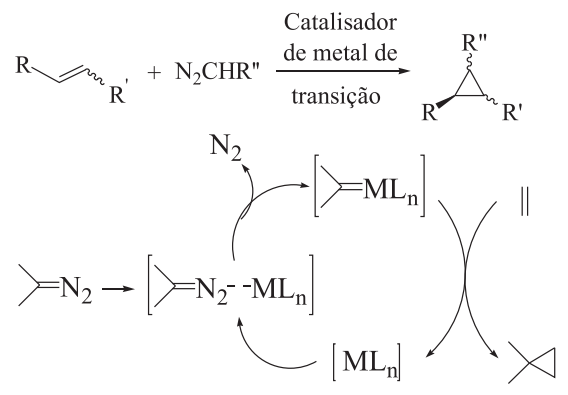

Esquema 4. Mecanismo da ciclopropanação com diazocompostos

Embora um grande número de sais de metais de transição interaja com o $\mathrm{RCH}=\mathrm{N}_{2}(\mathrm{Ni}, \mathrm{Pd}, \mathrm{Cu}, \mathrm{Fe}, \mathrm{Co}, \mathrm{Ru}, \mathrm{Zn}, \mathrm{U}, \mathrm{Os})$ para formar intermediários metalocarbenos ${ }^{29}$, apenas no início da década de 90 surgiu o primeiro processo relevante envolvendo a adição de diazoalcanos a olefinas sob condições de catálise assimétrica: Pfaltz mostrou que o complexo de $\mathrm{Cu}$ com a semicorrina quiral $\mathbf{1 2}$ catalisa a ciclopropanação do trans-cinamato de metila em moderada pureza óptica ${ }^{14,30}$ (Equação 8).

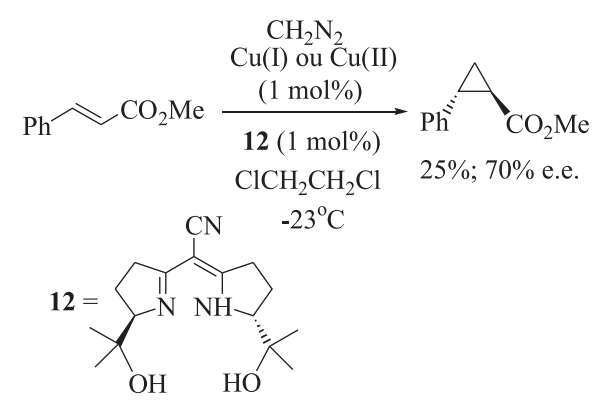

Somente 10 anos após, Charette e colaboradores ${ }^{31}$ obtiveram bons rendimentos químicos ao utilizarem o complexo entre o $\mathrm{Cu}(\mathrm{I})$ e a bis(oxazolina) quiral $\mathbf{1 3}$ na ciclopropanação do trans-cinamato de metila (Equação 9). 


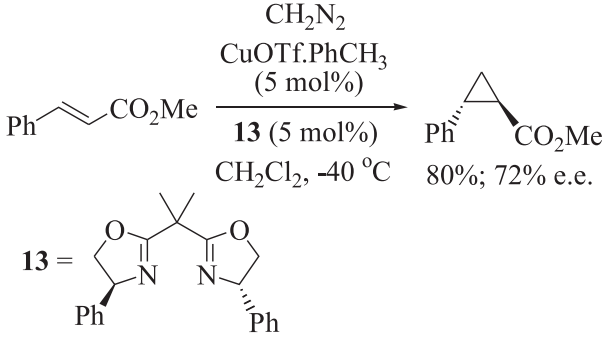

\section{CICLOPROPANAÇÃO COM $\alpha$-DIAZOÉSTERES}

A ciclopropanação de alcenos aquirais com $\alpha$-diazoésteres sob catálise homogênea com complexos quirais de metal de transição formando misturas de ésteres trans e cis-ciclopropânicos é um dos processos assimétricos mais largamente estudados e leva à formação simultânea de dois centros estereogênicos ${ }^{5,14,16}$ (Equação 10).

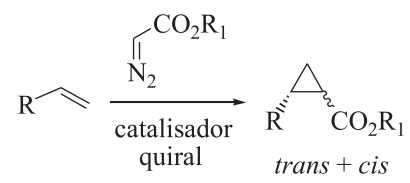

(quirais)

Muitos complexos quirais de metais de transição, como os derivados de $\mathrm{Cu}, \mathrm{Rh}, \mathrm{Ru}$ e $\mathrm{Co}$, catalisam esta reação e algumas conclusões gerais podem ser tiradas a partir das revisões recentes sobre o assunto ${ }^{5,14,16}$ : as maximizações tanto da diastereosseletividade (razão trans:cis) quanto da enantiosseletividade (\% e.e.) dependem não apenas da seleção do catalisador quiral (isto é, da escolha apropriada do sistema metal-ligante quiral), mas também do volume da porção alcoólica do $\alpha$-diazoéster ${ }^{5}$ : ésteres com porções alcoólicas volumosas produzem adutos em elevadas razões trans:cis; os catalisadores mais efetivos e utilizados para preparação de transciclopropanos são aqueles à base de $\mathrm{Cu}$. Catalisadores à base de $\mathrm{Rh}$ são muito reativos, mas geralmente produzem menores enantioe diastereosseletividades e, por isso, não são muito usados na ciclopropanação de $\alpha$-diazoésteres ${ }^{16}$; os catalisadores à base de $\mathrm{Ru}$ são muito eficientes, mas seu escopo é menor que aqueles baseados em $\mathrm{Cu}$. Por sua vez, os catalisadores à base de Co são geralmente usados em ciclopropanações cis-estereosseletivas e os ligantes quirais são usualmente complexos ${ }^{16}$.

Uma vez que excelentes revisões estão disponíveis para a ciclopropanação assimétrica com $\alpha$-diazoésteres ${ }^{5,14,16}$, serão enfocados apenas os catalisadores quirais derivados de metais de transição mais recentemente descritos e efetivos em termos das enantio- e diastereosseletividades.

\section{Catalisadores à base de cobre}

O primeiro exemplo de reação de ciclopropanação intermolecular assimétrica de olefinas com $\alpha$-diazoésteres usando catalisadores à base de $\mathrm{Cu}$ foi descrito por Nozaki ${ }^{32}$, em 1966. Desde então, um grande número de ligantes quirais foram sintetizados e avaliados nesta reação.

As bis(oxazolinas) constituem-se em uma das principais classes de ligantes quirais em síntese assimétrica ${ }^{33}$ e seus complexos de $\mathrm{Cu}(\mathrm{I})$ e $\mathrm{Cu}$ (II) são os catalisadores homogêneos mais populares e eficientes para a reação de ciclopropanação assimétrica de olefinas com $\alpha$ diazoésteres para gerar misturas enriquecidas em ésteres transciclopropânicos ${ }^{5,14,16}$. Alguns dos melhores resultados relatam o emprego das bis(oxazolinas) $\mathbf{1 4 - 2 2 ^ { 3 4 }}$ (Figura 5), onde as razões diastereoisoméricas (rd; razão trans: cis) e enantiosseletividades (\% e.e.) mostradas para cada ligante são relativas à reação do estireno com $\alpha$-diazoésteres ${ }^{35}$.

O complexo de $\mathrm{Cu}(\mathrm{I})$ da bis(oxazolina) 14, descrito por Evans ${ }^{34}$ na década de 90, devido a sua eficiência na ciclopropanação de ésteres volumosos (derivados do BHT), é ainda considerado o padrão ao qual novos ligantes bis(oxazolinas) são comparados. Em adição, as ciclopropanações do estireno com o $\alpha$-diazoacetato de etila na presença de líquidos iônicos como $[\mathrm{BMIN}]\left[\mathrm{BF}_{4}\right]$ e $[\mathrm{BMIM}]\left[\mathrm{PF}_{6}\right]$ ocorrem em altas enantiosseletividades ${ }^{34}$; estas espécies permitem uma fácil remoção do catalisador do meio reacional ao final da reação. A bis(oxazolina) $\mathbf{1 4}$ tem sido extensivamente empregada em ciclopropanações de diversos tipos de olefinas funcionalizadas, como sililenol-éteres, furanos e álcoois alílicos protegidos ${ }^{36}$. Os catalisadores quirais 15-19 também fornecem elevadas enantiosseletividades na ciclopropanação do diazoacetato volumoso derivado do $l$-mentol ${ }^{34}$; contudo, as reações a partir do diazoacetato de etila usando o ligante 20 também ocorrem em altas enantiosseletividades, mas com o comprometimento das razões trans: cis $^{34}$.

Mais recentemente, as aza-bis(oxazolinas) 21 e 22 foram descritas como ligantes para as reações catalisadas por $\mathrm{Cu}(\mathrm{II})^{34}$. Por sua vez, Pfaltz e colaboradores ${ }^{34}$ introduziram as bis(oxazolinas) aniônicas de estruturas 23 ("borabox") como ligantes altamente efetivos na formação de ésteres trans-ciclopropânicos em altas \% e. e.

As bis(oxazolinas) de estruturas $\mathbf{2 4}$ foram relatadas por Gibson e colaboradores $^{34}$ como sendo o único caso para a ciclopropanação cisestereosseletiva do estireno com diazoacetatos de etila e de terc-butila.

O desenvolvimento de ligantes perfluorados como 25 apresentam a vantagem de serem facilmente removidos do meio reacional por cromatografia em coluna gel de sílica ou por extração com solventes perfluorados ${ }^{34}$.
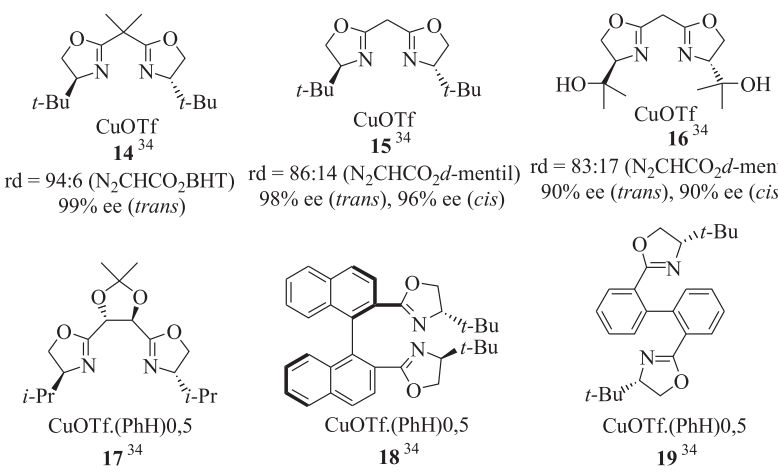
$\mathrm{rd}=86: 14\left(\mathrm{~N}_{2} \mathrm{CHCO}_{2} d\right.$-mentil)
$98 \%$ ee $($ trans $), 96 \%$ ee $($ cis $)$$\quad \begin{gathered}\mathrm{d}=83: 17\left(\mathrm{~N}_{2} \mathrm{CHCO}_{2} d \text {-mentil) }\right. \\ 90 \% \text { ee }(\text { trans }), 90 \% \text { ee }(\text { cis })\end{gathered}$

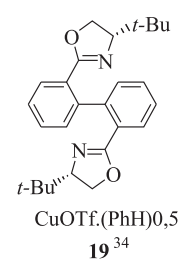

$\mathrm{rd}=85: 15\left(\mathrm{~N}_{2} \mathrm{CHCO}_{2} l\right.$-mentil) $\mathrm{rd}=68: 32\left(\mathrm{~N}_{2} \mathrm{CHCO}_{2} l\right.$-mentil $) \mathrm{rd}=81: 19\left(\mathrm{~N}_{2} \mathrm{CHCO}_{2} l\right.$-mentil $)$ $89 \%$ ee (trans), $89 \%$ ee (cis) $\quad 95 \%$ ee (trans), $97 \%$ ee (cis) $\quad 84 \%$ ee (trans), $92 \%$ ee (cis)
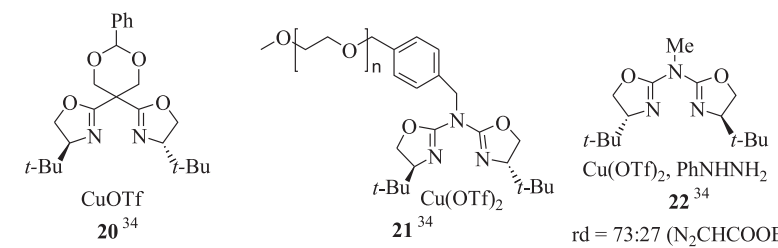

$\mathrm{rd}=67: 33\left(\mathrm{~N}_{2} \mathrm{CHCOOEt}\right)$

$\mathrm{rd}=71: 29\left(\mathrm{~N}_{2} \mathrm{CHCO}_{2} \mathrm{Me}\right)$

$\mathrm{rd}=73: 27\left(\mathrm{~N}_{2} \mathrm{CHCOOEt}\right)$

$99 \%$ ee (trans), $89 \%$ ee (cis)

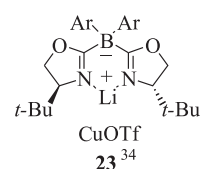

$23^{34}$

$\mathrm{rd}=99: 1\left(\mathrm{~N}_{2} \mathrm{CHCO}_{2} \mathrm{BHT}\right)$ $99 \%$ ee (trans)

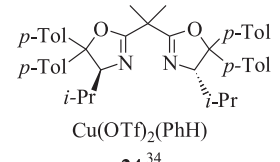

$24^{34}$

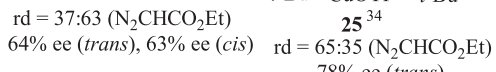

Figura 5. Bis(oxazolinas) na ciclopropanação catalisada por Cu(I) 
O mecanismo proposto para esta reação ${ }^{16,17}$ é mostrado no Esquema 5. A decomposição do complexo bis(oxazolina)- $\mathrm{Cu}(\mathrm{I}) \mathrm{A}$ leva à oxazolina $\mathbf{B}$ e ao catalisador ativo oxazolina- $\mathrm{Cu}(\mathrm{I}) \mathbf{C}$, que reage com o $\alpha$-diazoéster para formar o complexo catalisador-diazoéster D. A subseqüente eliminação de $\mathrm{N}_{2}$ leva ao intermediário carbeno$\mathrm{Cu}(\mathrm{I}) \mathbf{E}$, que reage com a olefina resultando no intermediário carbeno-Cu(I)-olefina $\mathbf{F}$. Nesta etapa ocorre a formação do anel ciclopropano no complexo quiral $\mathbf{G}$, que se decompõe ao éster ciclopropânico regenerando o catalisador ativo $\mathbf{C}$.

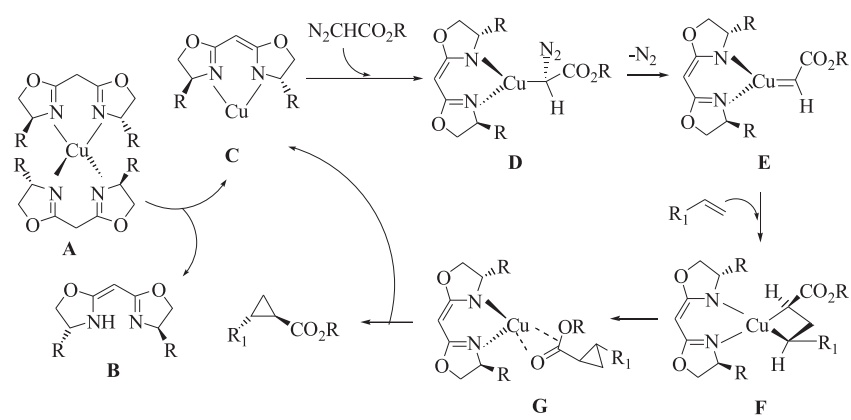

Esquema 5. O mecanismo da ciclopropanação catalisada por bis(oxazolina)$\mathrm{Cu}(\mathrm{I})$

A olefina ataca preferencialmente a face si do intermediário carbeno- $\mathrm{Cu}(\mathrm{I}) \mathbf{E}$, que é estericamente menos bloqueada pelo grupamento $\mathrm{R}^{37}$ (Esquema 6). A estereoquímica das ciclopropanações catalisadas por bis(oxazolinas)- $\mathrm{Cu}(\mathrm{I})$ tem sido racionalizada a partir de cálculos teóricos empregando a Teoria do Funcional de Densidade (DFT) e as energias relativas calculadas estão em concordância com as razões trans: cis e com as \% e.e. dos produtos ${ }^{37,38}$.

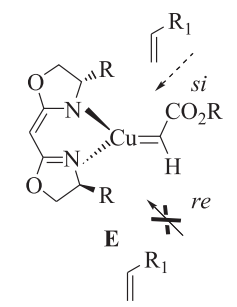

Esquema 6. A origem da estereosseletividade nas ciclopropanações com bis(oxazolinas)- $\mathrm{Cu}(\mathrm{I})$

Alguns exemplos de ciclopropanação enantiosseletiva intramolecular de $\alpha$-diazocetonas com catalisadores de bis(oxazolinas)- $\mathrm{Cu}(\mathrm{I})$ têm sido relatados ${ }^{39}$. Shibasaki et $a l^{40}$ descreveram a síntese dos anéis $\mathrm{C}$ e $\mathrm{D}$ do phorbol através da ciclopropanação assimétrica intramolecular de silil-enol-éter 26 (Equação 11). Após otimização, a biciclocetona 27 foi obtida em $70 \%$ rendimento e $92 \%$ de excesso enantiomérico quando $15 \mathrm{~mol}$ $\%$ da bis(oxazolina) 28 e 5 mol \% de CuOTf foram usados na formação do catalisador quiral.

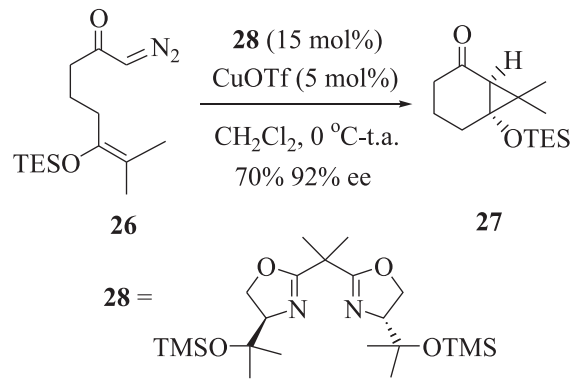

Outras classes de ligantes quirais têm sido empregadas nas reações de ciclopropanação assimétrica de olefinas com $\alpha$ diazoésteres sob condições homogêneas (Figura 6). Os ligantes do tipo semicorrina ent-12 e $\mathbf{2 9}^{41}$, devido às suas baixas acidez de Lewis, levam a baixos rendimentos nos ciclopropanos de alcenos desativados. Por sua vez, muitas vezes os ligantes derivados de bipiridinas como $\mathbf{3 0} \mathbf{3}^{42}$, bem como as diaminas $\mathbf{3 1} \mathbf{1}^{43}$ e $\mathbf{3 2}^{44}$ produzem bons resultados nessas reações. O uso do bis-aza-ferroceno 33 origina enatiosseletividades muito altas não apenas com estireno, mas também com alcenos monossubstituídos ${ }^{45}$.

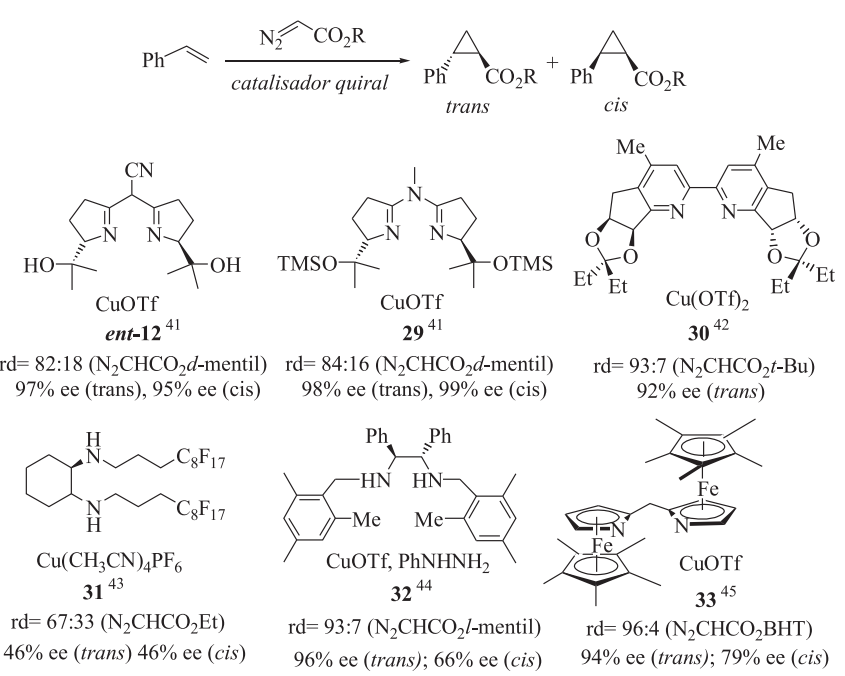

* Para cada ligante são mostradas as razões trans: cis e as \% e. e. nas ciclopropanações.

Figura 6. Outros ligantes na ciclopropanação catalisada por $\mathrm{Cu}$

\section{Catalisadores à base de rutênio}

Em geral, os catalisadores quirais à base de Ru são menos reativos que aqueles derivados de $\mathrm{Cu}$ e $\mathrm{Rh}$. Muitos deles convertem alcenos aril-substituídos aos seus correspondentes ciclopropanos, mas menores rendimentos são observados com alcenos substituídos por grupos alquila ${ }^{16}$.

$\mathrm{O}$ primeiro catalisador efetivo à base de $\mathrm{Ru}$ foi descrito por Nishiyama et al. ${ }^{46}$, em 1994: o ligante bis(oxazolina) Pybox-i-Pr reage com o $\mathrm{RuCl}_{2}$ ( $p$-cimeno) levando ao catalisador $(R, R)$-trans$\mathrm{Cl}_{2} \mathrm{Ru}$ (pybox-ip) $\left(\mathrm{H}_{2} \mathrm{C}=\mathrm{CH}_{2}\right), \mathbf{R u}$ (ip-Pybox) (34), isolado como seu complexo com eteno. Este catalisador é eficiente na ciclopropanação do estireno para formar o trans-ciclopropano correspondente em elevadas razões diastereoisoméricas (rd) trans: cis e \% e. e. (Figura 7).

O sistema catalítico Ru-pybox é, provavelmente, o mais estudado para a ciclopropanação baseada em $\mathrm{Ru}$ e muitas variações estruturais do ligante pybox foram empregadas ${ }^{16,46}$. Por exemplo, o catalisador Ru-35, que incorpora uma unidade polimérica ao pybox, foi introduzido recentemente por Mayoral e colaboradores ${ }^{46}$. O catalisador Ru-Pybox (36), que não apresenta um eixo $\mathrm{C}_{2}$, catalisa eficientemente a reação produzindo o derivado ciclopropânico não apenas em altas enantiosseletividades, mas também com melhor razão trans: cis $^{47}$. Foi racionalizado que a remoção de um dos substituintes do sistema oxazolina cria mais espaço para aproximação do grupo éster. Outras variações no sistema pybox incluem a presença de anel tiofeno no $\mathbf{R u - T i o b o x ~}(\mathbf{3 7})^{48}$ e a modificação no sistema oxazolina contido no catalisador Ru-Tiopybox $(\mathbf{3 8})^{49}$, ambos menos efetivos que os catalisadores de Nishiyama 35 e 36. Posteriormente este autor descreveu novos catalisadores Ru-pybox 39 contendo grupos polares no anel bis(oxazolina) para a 
ciclopropanação assimétrica de alcenos em meios aprótico e/ ou bifásico $\left(\mathrm{THF}-\mathrm{H}_{2} \mathrm{O} \text {, tolueno- } \mathrm{H}_{2} \mathrm{O} \text {, tolueno- } i-\mathrm{PrOH}\right)^{50}$.

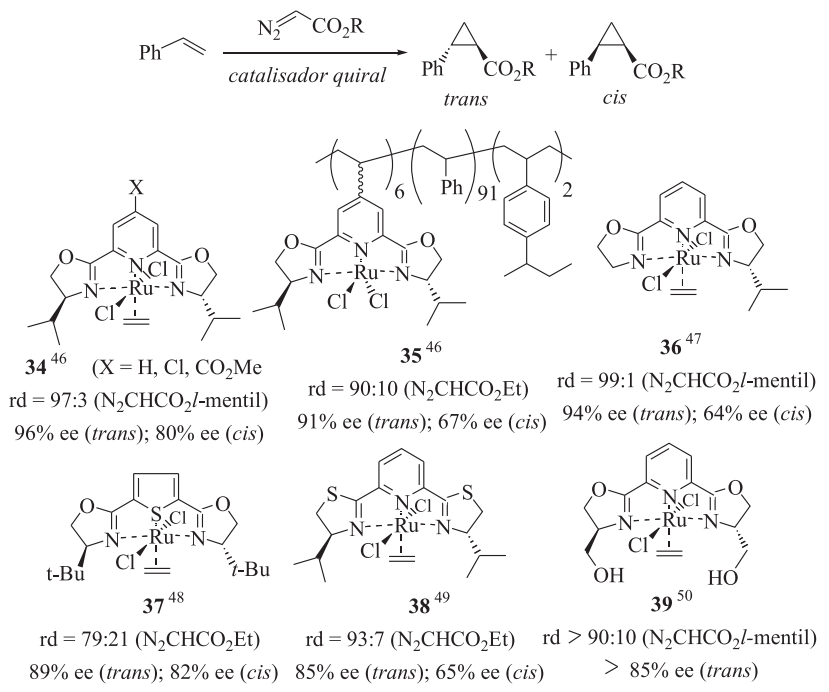

Figura 7. Catalisadores Ru-pybox para ciclopropanação assimétrica

O sentido da indução assimétrica na reação do estireno com $\alpha$ diazoésteres empregando o catalisador de Nishiyama Ru(ip-Pybox) (34) é consistente com o modelo mostrado na Figura 8, onde o alceno ataca de forma que coloque o grupo fenila afastado do grupo éster do $\alpha$-diazocomposto e da porção isopropila do catalisador ${ }^{46}$.

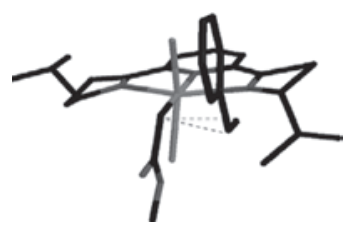

Figura 8. Modelo do estado de transição para ciclopropanação com Ru (ip-pybox)

O catalisador de Nishiyama Ru(ip-Pybox) (34) tem sido empregado em ciclopropanações durante as sínteses totais de substâncias biologicamente ativas ${ }^{51,52}$. Um exemplo recente é a ciclopropanação assimétrica de 1-tosil-3-vinil-indóis substituídos com o $\alpha$ diazoacetato de etila para produzir o éster indol-ciclopropânico correspondente (trans: cis $=90: 10 ; 86 \%$ e.e. $)^{52}$ (Esquema 7). Esta substância foi empregada na síntese da homotriptamina BMS-505130, que é um inibidor seletivo da recaptação do neurotransmissor serotonina no sistema nervoso central.

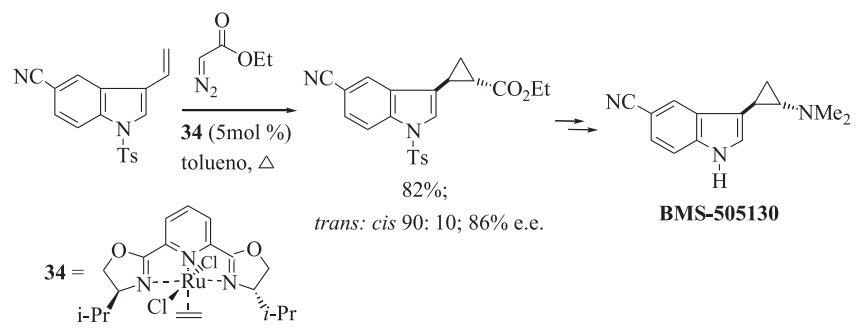

Esquema 7. Catalisador Ru(ip-Pybox) 34 na síntese do BMS-505130

Muitos outros complexos quirais de rutênio foram descritos e alguns deles são excelentes catalisadores na ciclopropanação do estireno com $\alpha$-diazoésteres (Figura 9). Os catalisadores Ru-porfirinas quirais
$\left[\mathrm{Ru}\left(\mathrm{P}^{*}\right)(\mathrm{CO})\right]$ produzem excelentes níveis de diastereo- e enantiocontroles na produção de ésteres trans-ciclopropânicos ${ }^{53}$. Alguns exemplos incluem os complexos [Ru(tetrarilporfirina)(CO)] 40 e 41 ${ }^{54}$. Complexos de Ru com bases de Schiff quirais, como 42, também produzem excelentes seletividades de trans-ciclopropanos ${ }^{55}$. Poucos catalisadores à base de Ru exibem seletividade cis em reações de ciclopropanação ${ }^{56}$, onde os mais eficientes são os derivados de ligantes PNNP, como o catalisador de Mezzetti $43^{57}$ e os cátions de 16 elétrons $[\mathrm{RuCl}(\mathrm{PNNP})]^{+}$como 44${ }^{58}$. Os complexos Ru-salen, 45 e 46, em que dois dos sítios coordenantes estão ocupados pelo ligante piridina, dão excelentes excessos enantioméricos nas ciclopropanações do estireno e do acrilato de metila, respectivamente ${ }^{59}$.

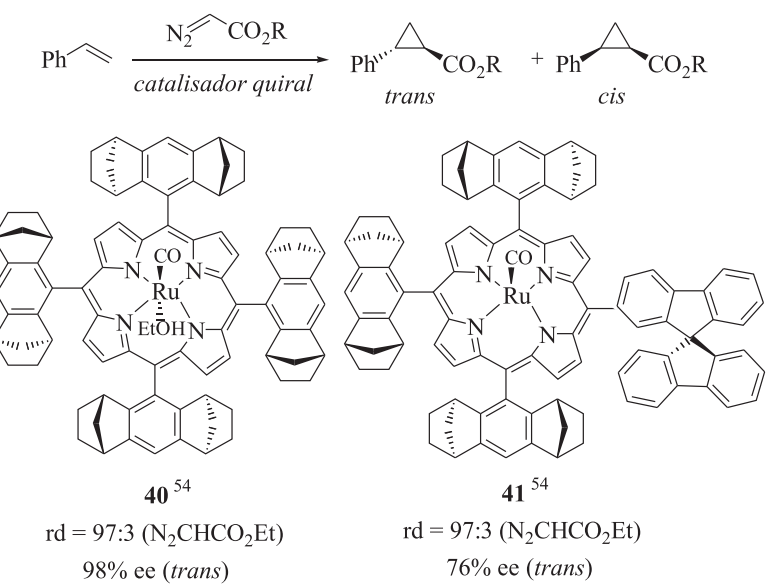

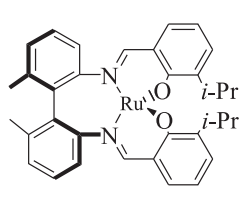

$42^{55}$

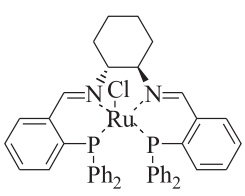

$43^{57}$

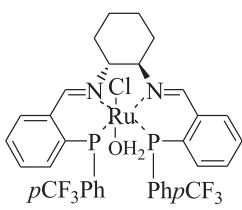

$44^{58}$
$95 \%$ ee (trans) $\mathrm{rd}=2: 98\left(\mathrm{~N}_{2} \mathrm{CHCO}_{2} \mathrm{Et}\right)$

$80 \%$ ee (cis)
99\% ee (cis)

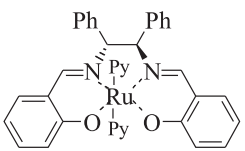

$45^{59}$

$\mathrm{rd}=77: 23\left(\mathrm{~N}_{2} \mathrm{CHCO}_{2} \mathrm{Et}\right)$

99\% ee (trans)

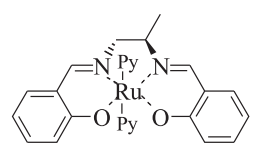

$46^{59}$

$\mathrm{rd}=99: 1\left(\mathrm{~N}_{2} \mathrm{CHCO}_{2} \mathrm{Et}\right)$ 95\% ee (trans)

* Para cada ligante são mostradas as razões trans: cis e as \% e. e. nas ciclopropanações.

Figura 9. Ciclopropanação do estireno catalisada por rutênio

\section{Catalisadores à base de cobalto}

Complexos de Co(II) são catalisadores efetivos para a decomposição de $\alpha$-diazoésteres aos respectivos carbenos, que convertem alcenos aos trans-ciclopropanos em excelentes diastereo- e enantiosseletividades ${ }^{16}$. Yamada mostrou que complexos 3oxobutilidenoaminatocobalto (II), tal como 47, são efetivos na ciclopropanação trans-estereosseletiva do estireno com $\alpha$ diazoésteres na presença de N-metilimidazola (NMI) para aumentar não somente a velocidade da reação, mas também a enantiosseletividade $^{60}$ (Figura 10). Katsuki desenvolveu uma série de ligantes quirais para a ciclopropanação trans-estereosseletiva de alcenos; dentre eles, o complexo Co(II)(salen) 48 mostrou-se bastante efetivo para a decomposição do diazoacetato de $t$-butila na presença de estireno para formar o respectivo trans-ciclopropano 
em excelentes diastereo- e enantiosseletividades ${ }^{61}$. Katsuki mostrou que catalisadores de Co baseados no arcabouço quiral do salen, como 49, são efetivos para a ciclopropanação cis-estereosseletiva ${ }^{62}$.

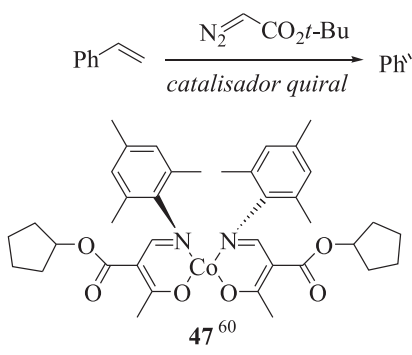

$\mathrm{rd}=91: 9 ; 96 \%$ ee (trans)

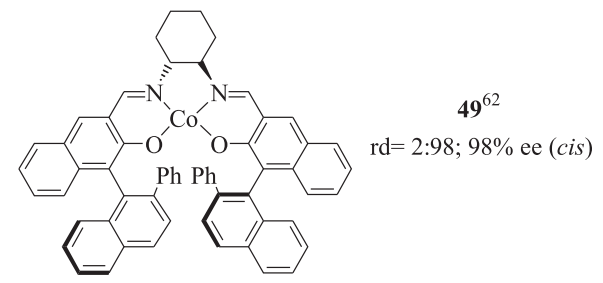

* Para cada ligante são mostradas as razões trans: cis e as \% e. e. nas ciclopropanações.

Figura 10. Catalisadores à base de Co em ciclopropanação assimétrica

Posteriormente Katsuki mostrou que, embora os catalisadores de cobalto e rutênio $\mathrm{Co}(\mathrm{II})$ (salen) $\mathbf{5 0}$ e $\left(\mathrm{ON}^{+}\right) \mathrm{Ru}(\mathrm{II})$ (salen) $\mathbf{5 1}$ forneçam ciclopropanações altamente cis-diastereosseletivas, eles levam a induções assimétricas com sentidos opostos, a despeito de apresentarem o mesmo ligante salen em suas estruturas ${ }^{63}$ (Esquema 8).
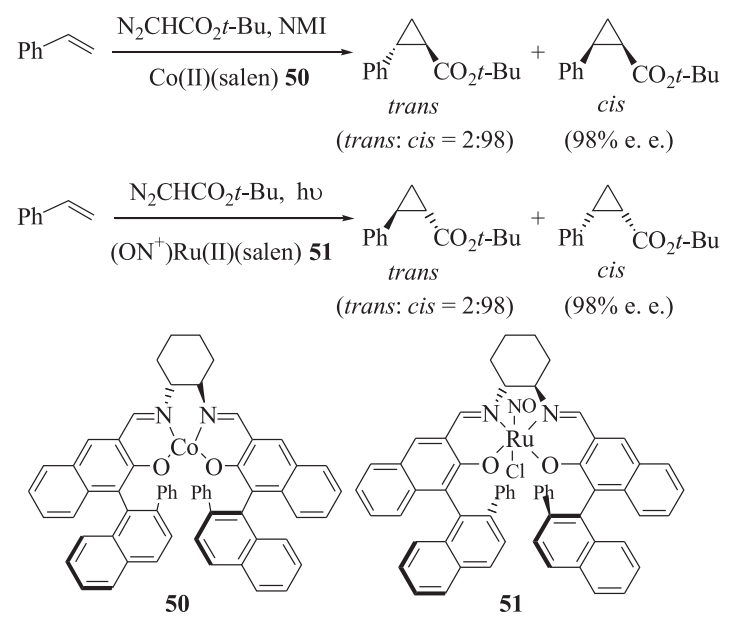

Esquema 8. Co(II)(salen) e $(O N+) R u(I I)($ salen $):$ indução assimétrica oposta

Recentemente os complexos Co-porfirina foram introduzidos como catalisadores na reação de ciclopropanação assimétrica do estireno (Figura 11). Enquanto o complexo de Co do derivado 52 da vitamina $\mathrm{B}_{12}$ produz adutos $\mathrm{cis}^{64}$, os catalisadores de Co derivados de $\mathbf{5 3}$ e de $\mathbf{5 4}$ levam ao isômero $\operatorname{trans}^{65,66}$.

\section{Catalisadores à base de ródio}

Os catalisadores à base de ródio têm sido largamente empregados na reação de ciclopropanação assimétrica inter- e intramolecular de alcenos ${ }^{16}$. A maior parte dos catalisadores quirais à base de ródio até hoje desenvolvidos pertencem a duas classes (Figura 12): os

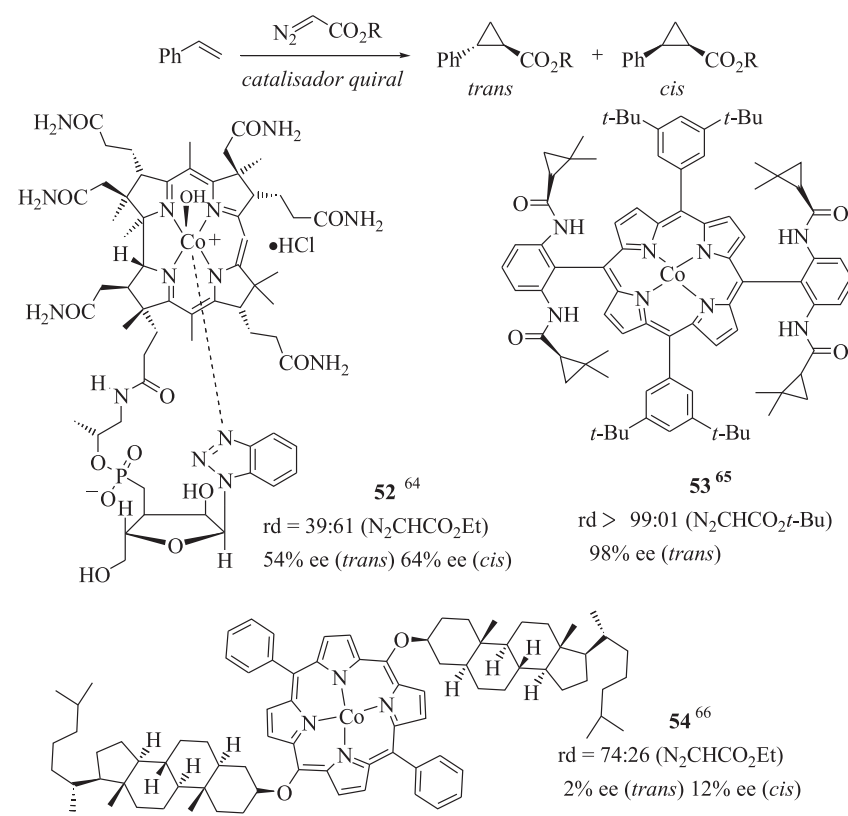

* Para cada ligante são mostradas as razões trans: cis e as \% e. e. nas ciclopropanações.

Figura 11. Complexos Co-porfirinas em ciclopropanação

carboxilatos de di-Rh(II) de fórmula geral $\mathrm{Rh}_{2}\left(\mathrm{O}_{2} \mathrm{CR}\right)_{2}\left(\mathrm{~L}^{*}\right)_{2}$, como os compostos 55-57 $57-69$ e os carboxamidatos de di-Rh(II) de fórmula geral $\mathrm{Rh}_{2} \mathrm{~L}_{4}{ }^{*}$, como os compostos 58-62 $\mathbf{2}^{70,71}$.

Carboxilatos de di-Rh (II): $\mathbf{R h}_{2}\left(\mathrm{O}_{2} \mathrm{CR}\right)_{2}\left(\mathrm{~L}^{*}\right)_{2}$

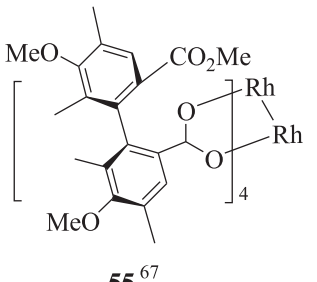<smiles>[R]C1(C)OC(C(Cc2ccccc2)N2C(=O)c3ccccc3C2=O)OC1(C)C</smiles>

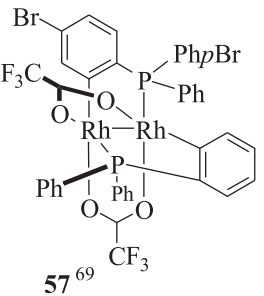

Carboxamidatos de di-Rh (II): $\mathbf{R h}_{2} \mathbf{L}_{4}^{*}$

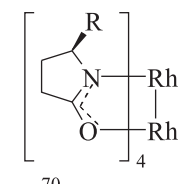

58: ${ }^{70} \mathrm{R}=\mathrm{COOMe}$

$\mathrm{Rh}_{\mathbf{2}}(\mathbf{5 S} \text {-MEPY })_{4}$

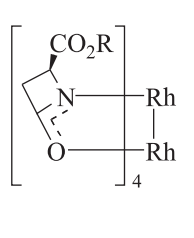

$$
\begin{aligned}
& \text { 59: }{ }^{71} \mathrm{R}=i \text {-Bu } \mathbf{R h}_{\mathbf{2}}(\mathbf{4 S} \text {-IBAZ) })_{4} \\
& 60::^{71} \mathrm{R}=\mathrm{Me} \quad \mathbf{R h}_{\mathbf{2}}\left(\mathbf{4} S \text {-MEAZ) }{ }_{4}\right. \\
& \text { 61: }{ }^{71} \mathrm{R}=\mathrm{Bn} \quad \mathbf{R h}_{2}\left[(3 S)-b^{-b n a z}\right]_{4} \\
& \text { 62: }{ }^{71} \mathrm{R}=m \\
& i \text {-Pr }
\end{aligned}
$$

Figura 12. Principais tipos de catalisadores de Rh em ciclopropanação assimétrica

A razão trans: cis na ciclopropanação de olefinas em reações catalisadas por $\mathrm{Rh}(\mathrm{II})$ não é alta, mesmo quando $\alpha$-diazoésteres estericamente impedidos são empregados ${ }^{16}$. Essa importante desvantagem em relação aos catalisadores à base de $\mathrm{Cu}, \mathrm{Ru}$ e Co tem minimizado o uso de catalisadores de $\mathrm{Rh}$ (II) em processos intermoleculares envolvendo $\alpha$-diazoésteres simples. Os exemplos mostrados na Tabela 2 mostram que, embora o nível de enantiosseleção seja algumas vezes excelente, o diastereocontole não é muito bom.

Mais recentemente complexos de $\mathrm{Rh}(\mathrm{III})$ com ligantes porfirina de simetria $D_{4}$ denotados por $\left[\mathrm{Rh}\left(\mathrm{P}^{*}\right)(\mathrm{I})\right]$, como 63 , foram introduzidos em reações de ciclopropanação assimétrica de olefinas; con- 


$$
\underset{\begin{array}{c}
\text { catalisador } \\
\text { quiral }
\end{array}}{\stackrel{\mathrm{N}_{2}}{\mathrm{CO}_{2} \mathrm{R}}} \overbrace{\text { trans: cis }}^{\mathrm{CO}_{2} \mathrm{R}}
$$

Tabela 2. Ciclopropanação enantiosseletiva catalisada por $\mathrm{Rh}(\mathrm{II})$

\begin{tabular}{lcccc}
\hline cat. & $\mathrm{R}$ & $\%$ & trans:cis & $\begin{array}{c}\% \text { e. e. } \\
\text { (trans;cis) }\end{array}$ \\
\hline 59 & $c$-Hex2CH & 81 & $46: 54$ & $66 ;>98$ \\
55 & $d$-Mantil & 100 & $37: 63$ & $45 ; 99$ \\
58 & $d$-Mntil & 100 & $57: 43$ & $24 ; 91$ \\
57 & $\mathrm{Et}$ & 60 & $55: 45$ & $86 ; 87$ \\
62 & $\mathrm{t}-\mathrm{Bu}$ & 47 & $74: 26$ & $71 ; 94$ \\
\hline
\end{tabular}

tudo as baixas razões trans: cis características do uso de

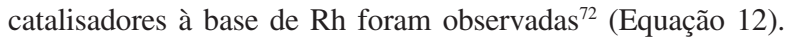

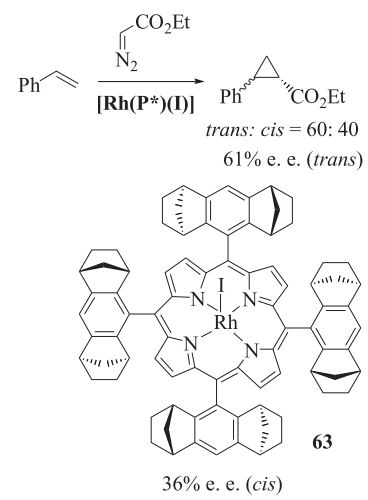

Os diazocompostos derivados de substâncias dicarboniladas são substancialmente menos reativos que os $\alpha$-diazoésteres ${ }^{16}$. Assim, é necessário o emprego de catalisadores muito ativos para gerar os intermediários metalocarbenos. Por exemplo, o $\mathrm{Rh}_{2}[(4 S) \mathrm{MEAZ}]_{4} 60$ catalisa a ciclopropanação do estireno com o diazomalonato de dimetila em $97 \%$ de rendimento, mas com apenas $50 \%$ e. e. ${ }^{73}$ (Esquema 9). Mais recentemente, Muller e colaboradores introduziram ilídeos de feniliodônio como substitutos de diazocompostos derivados de substâncias dicarboniladas na reação de ciclopropanação do estireno catalisada pelo carboxilato de di-Rh(II) $\mathbf{R h}_{2}[(\boldsymbol{S}) \text {-nttl }]_{4} \mathbf{6 4}^{74}$. Contudo, as enantiosseletividades permaneceram insatisfatórias, a despeito dos bons rendimentos químicos. Ademais, o uso de ilídeos de feniliodônio apresenta a desvantagem de gerar PhI como subproduto, ao passo que os diazocompostos produzem nitrogênio.

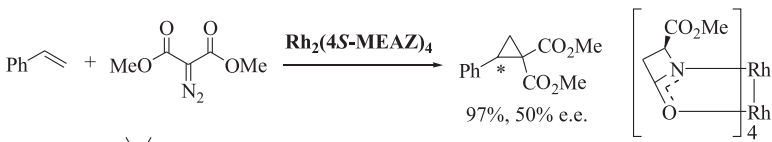

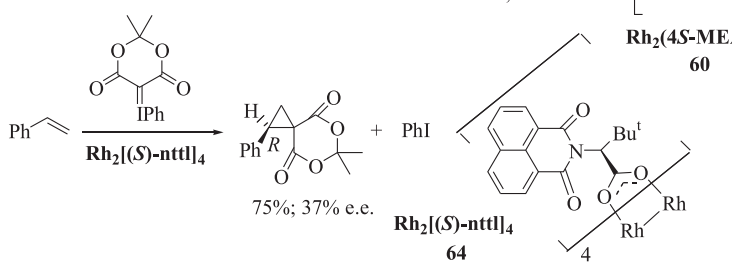

Esquema 9. Ciclopropanação assimétrica com $\beta$-dicarbonilados

Outros catalisadores quirais à base de Rh menos importantes são descritos na literatura para a ciclopropanação assimétrica de alcenos com diazoésteres na formação de complexos ciclopropânicos altamente substituídos ${ }^{75}$.

\section{CARBOIDRATOS EM CICLOPROPANAÇÃO ASSIMÉTRICA}

Um dos mais importantes avanços na reação de ciclopropanação assimétrica na última década envolve o uso de carboidratos como matérias-primas quirais empregando a abordagem do "chiral pool" 76,77 . A ciclopropanação de glicais produz estruturas bicíclicas únicas, que combinam a elevada reatividade dos ciclopropanos associada à extrema pureza óptica e a elevada funcionalização dos derivados de carboidratos.

A reação de ciclopropanação de Simmons-Smith empregando a modificação de Furukawa (uso de $\mathrm{Et}_{2} \mathrm{Zn}$ em lugar de $\mathrm{Zn}-\mathrm{Cu}$ ) apresenta resultados reprodutíveis em condições reacionais brandas ${ }^{78}$. Estas condições permitiram elevados rendimentos e controles estereoquímicos na conversão de $\mathbf{6 5}$ em $\mathbf{6 6}^{78}$ (Equação 13).

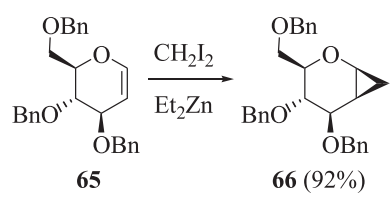

Elevadas diastereosseletividades também são observadas em ciclopropanações assimétricas de glicais protegidos, como 67 e 68 , com $\alpha$-diazoésteres para gerar os respectivos ciclopropanos 69 e 70 , tanto sob catálise de $\mathrm{Cu}(\mathrm{I})$ quanto de $\mathrm{Rh}(\mathrm{II})^{79}$ (Equações 14, 15).

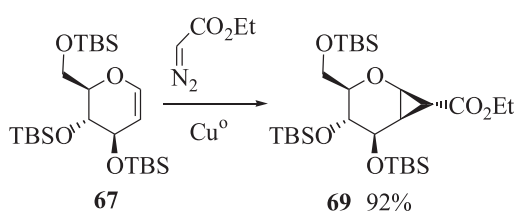

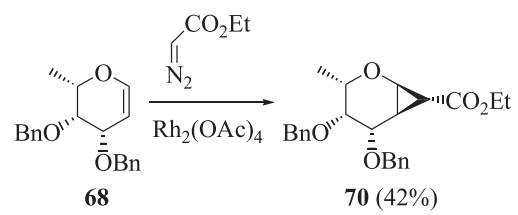

Olefinas portadoras de substituintes eletroatratores sofrem ciclopropanações diastereosseletivas com complexos glicosilidenos para dar espirociclopropanos substituídos, conforme é ilustrado para a converão do reagente quiral cromo manofuranosilideno $\mathbf{7 1}$ ao éster ciclopropânico 72 (Equação 16) ${ }^{80}$.

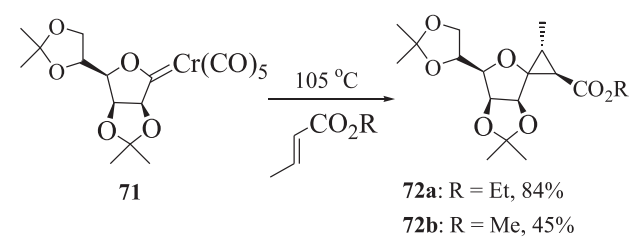

Embora derivados de carboidratos sejam bastante empregados em síntese assimétrica como auxiliares de quiralidade nas reações de DielsAlder $^{81}$, alfa-alquilação de enolatos ${ }^{82}$, aldol e adição conjugada ${ }^{83}$ e como ligantes quirais na reação de Reformatsky ${ }^{84}$, poucos trabalhos são descritos na literatura referentes ao emprego de derivados de carboidratos como ligantes quirais em ciclopropanações de olefinas com $\alpha$ diazoésteres sob condições de catálise assimétrica.

A base de Schiff $\mathbf{7 3}$ e os diaminocompostos $\mathbf{7 5}$ e $\mathbf{7 7}$ derivados da $\alpha$-D-glicose, bem como os ligantes 74 e 76 derivados da $\alpha$-Dmanose, formam quelatos com $\mathrm{Cu}(\mathrm{I})$ que catalisam a ciclopropanação do estireno com o $\alpha$-diazoacetato de etila levando ao res- 
pectivo éster ciclopropânico ${ }^{85}$ (Figura 13). A natureza do carboidrato e dos quelatos é crucial na determinação da enantiosseletividade da reação: as diiminas e diaminas derivadas da $\alpha$-D-glicose favorecem enantiômeros diferentes.

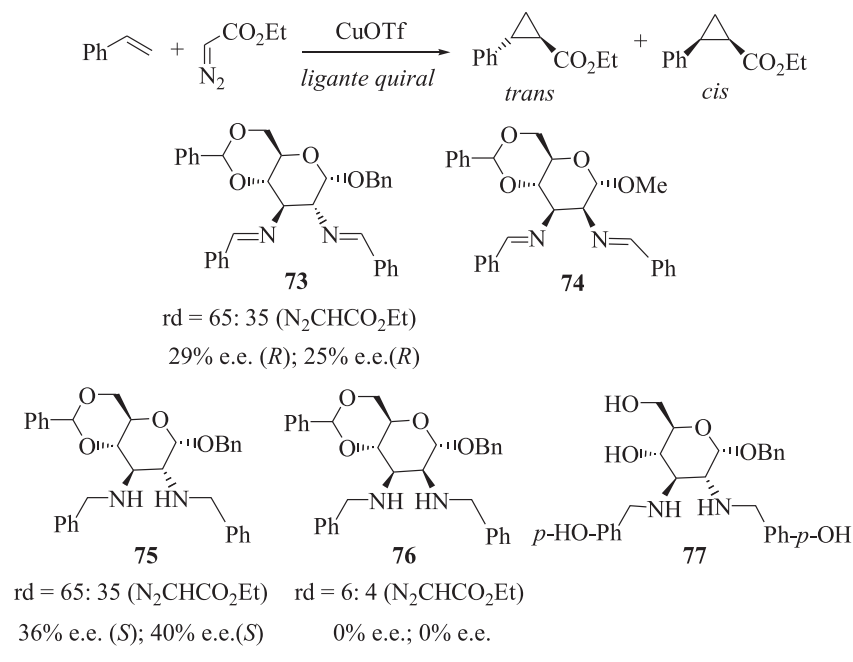

* Para cada ligante são mostradas as razões trans: cis e as \% e. e. nas ciclopropanações.

Figura 13. Carboidratos na ciclopropanação catalisada por $\mathrm{Cu}(\mathrm{I})$

Catalisadores de $\mathrm{Cu}(\mathrm{I})$ preparados a partir de bases de Schiff 78-81 derivadas de aminoaçúcares naturais têm sido aplicados na síntese de carboxilatos ciclopropânicos por meio da ciclopropanação assimétrica catalítica de haloalcenos ${ }^{85}$ (Figura 14). Esta reação ocorre em baixos a moderados rendimentos químicos e em baixos controles das diastereo- e enantiosseletividades, com um máximo de $58 \%$ e. e. ${ }^{86}$.

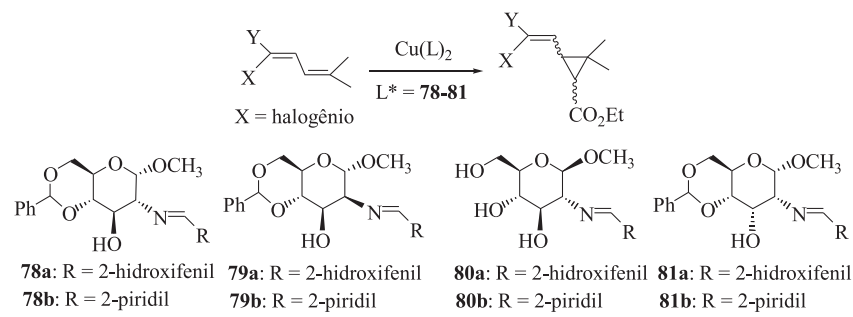

Figura 14. Aminocarboidratos na ciclopropanação catalisada por $\mathrm{Cu}(\mathrm{I})$

\section{CONCLUSÕES}

Um expressivo número de sistemas catalíticos foi desenvolvido para a reação de ciclopropanação assimétrica de olefinas, onde a combinação dos métodos descritos permite, em alguns casos, um controle eficiente da estereosseletividade da reação. Embora a reação de Simmons-Smith e o uso de diazoalcanos sejam processos estereoespecíficos, onde as configurações relativas dos substituintes no anel ciclopropânico encontram-se latentes nas olefinas de partida, os desenvolvimentos de reações cis-estereosseletivas e das ciclopropanações de olefinas não funcionalizadas são perspectivas interessantes. Uma vez que os maiores avanços na área são decorrentes dos empregos de $\alpha$-diazoacetatos como fontes de carbenos, esta metodologia tem sido aquela que permite melhores estereosseletividades em reações de ciclopropanação. De fato, a combinação dos diferentes ligantes quirais à base de $\mathrm{Cu}(\mathrm{I}), \mathrm{Ru}(\mathrm{II})$ e $\mathrm{Co}$ (II) possibilita os acessos tanto a derivados trans-ciclopropânicos quanto aos isômeros cis em elevadas eantiosseletividades.
Em todas as abordagens, algumas perspectivas interessantes consistiriam nos empregos de olefinas com diferentes graus de funcionalização, no incremento de compostos $\alpha$-dicarbonilados como fontes de carbeno e no desenvolvimento de ligantes quirais a partir de produtos naturais abundantes. Ademais, em que pese os empregos recentes de líquidos iônicos e de ligantes perfluorados para a remoção do catalisador ao final da reação, o desenvolvimento de métodos de ciclopropanação sob catálise assimétrica heterogênea continua um tema em aberto.

\section{REFERÊNCIAS}

1. Wong, H. N. C.; Hon, M. -T; Tse, C. -W; Ypi, Y. -C; Tanko, J.; Hudlicky, T.; Chem. Rev. 1989, 89, 165; Wurz, R. P.; Charette, A. B.; Org. Lett. 2005, 7, 2313.

2. Rappoport, Z., ed.; The Chemistry of the Cyclopropyl Group, Wiley: Chichester, 1997.

3. Herbert, R. B.; The Biosynthesis of Secondary Metabolites, $2^{\text {nd }}$ ed.; Chapman and Hall: London, 1988, p. 78.

4. Naumann, K. Em Chemistry of Plant Protection, Synthetic Pyrethroid Insecticides; Haug, G.; Hoffmann, H., eds.; Springer-Verlag: Heidelberg, 1990, vol. 5, p. 63; Arlt, D.; Jautelat, M.; Lantzsch, R.; Angew. Chem., Int. Ed. 1981, 20, 703.

5. Donaldson, W. A.; Tetrahedron 2001, 57, 8589.

6. Zheng, G. -C.; Hatano, M.; Ishitsuka, M. O.; Kusumi, T.; Kakisawa, H.; Tetrahedron Lett. 1990, 31, 2617; Zheng, G. -C.; Ichikawa, A.; Ishitsuka, M. O.; Kusumi, T.; Yamamoto, H.; Kakisawa, H.; J. Org. Chem. 1990, 55, 3677.

7. Niwa, H.; Wakamatsu, K.; Yamada, K.; Tetrahedron Lett. 1989, 30, 4543.

8. Seo, Y.; Cho, K. W.; Rho, J. -R.; Shin, J.; Kwon, B. M.; Bok, S. -H.; Song, J. -I.; Tetrahedron 1996, 52, 10583.

9. Gerwick, W. H.; Proteau, P. J.; Nagle, D. G.; Hamel, E.; Blokhin, A.; Slate, D. L.; J. Org. Chem. 1994, 59, 1243.

10. Graham, D. W.; Ashton, W. T.; Barash, L.; Brown, J. E.; Brown, R. D.; Canning, L. F.; Chen, A.; Springer, J. P.; Rogers, E. F.; J. Med. Chem. 1987, 30,1074 .

11. Zhang, X.; Hodgetts, K.; Rachwal, S.; Zhao, H.; Wasley, J. W. F.; Craven, K.; Brodbeck, R.; Kieltyka, A.; Hoffman, D.; Bacolod, M. D.; Girard, B.; Tran, J.; Thurkauf, A.; J. Med. Chem. 2000, 43, 3923; Yamaguchi, K.; Kazuta, Y.; Abe, H.; Matsuda, A.; Shuto, S.; J. Org. Chem. 2003, 68, 9255.

12. Suckling, C. J.; Angew. Chem., Int. Ed. 1988, 27, 537.

13. Salaün, J.; Chem. Rev. 1989, 89, 1247.

14. Seyden-Penne, J.; Chiral Auxiliaries and Ligands in Asymmetric Synthesis, John Wiley \& Sons, Inc.: New York, 1995, p. 489; Noyori, R.; Asymmetric Catalysis in Organic Synthesis, John Wiley \& Sons, Inc.: New York, 1994, p. 199; Doyle, M. P. Em Catalytic Asymmetric Synthesis; Ojima, I., ed.; Wiley-VCH: New York, 1993, Chap. 3; Pfaltz, A. Em Comprehensive Asymmetric Catalysis, Jacobsen, E. N.; Pfaltz, A.; Yamamoto, H., eds.; Springer: Berlin, 1999, vol. II, p. 513; Charette, A. B.; Lebel, H. Em Comprehensive Asymmetric Catalysis, Jacobsen, E. N.; Pfaltz, A.; Yamamoto, H., eds.; Springer: Berlin, 1999, vol. II, p. 581; Lydon, K. M.; McKervey, M. A. Em Comprehensive Asymmetric Catalysis; Jacobsen, E. N.; Pfaltz, A.; Yamamoto, H., eds.; Springer: Berlin, 1999, vol. II, p. 540.

15. Yu, M.; Pagenkopf, B. L.; Tetrahedron 2005, 61, 321.

16. Lebel, H.; Marcoux, J. -F.; Molinaro, C.; Charette, A. B.; Chem. Rev. 2003, 103, 977; Rechavi, D.; Lemaire, M.; Chem. Rev. 2002, 102, 3467.

17. Laroche, M.-F.; Belotti, D.; Cossy, J.; Org. Lett. 2005, 7, 171; Kim, H. Y.; Lurain, A. E.; Garcia-Garcia, P.; Carroll, P. J.; Walsh, P. J.; J. Am. Chem. Soc. 2005, 127, 13138; Fournier, J.-F.; Mathieu, S.; Charette, A. B.; J. Am. Chem. Soc. 2005, 127, 13140; Katagiri, T.; Iguchi, N.; Kawate, T.; Takahashi, S.; Uneyama, K.; Tetrahedron: Asymmetry 2006, 17, 1157.

18. Para estudos teóricos da reação de Simmons-Smith, ver: Frang, W.-H.; Phillips, D. L.; Wang, D.-Q.; Li, Y.-L.; J. Org. Chem. 2002, 67, 154; Hermann, H.; Lohrenz, J. C. W.; Kühn, A.; Boche, G.; Tetrahedron 2000, $56,4109$.

19. Takahashi, H.; Yoshioka, M.; Ohno M.; Kobayashi, S.; Tetrahedron Lett. 1992, 33, 2575; Takahashi, H.; Yoshioka, M.; Shibasaki, M.; Ohno M.; Imai, N.; Kobayashi, S.; Tetrahedron 1995, 51, 12013.

20. Denmark, S. E.; O'Connor, S. P.; Wilson, S. R.; Angew. Chem., Int. Ed. 1998, 37, 1149; Denmark, S. E.; O’Connor, S. P.; J. Org. Chem. 1997, 62, 584; Denmark, S. E.; O'Connor, S. P.; J. Org. Chem. 1997, 62, 3390; Denmark, S. E.; Christenson, B. L.; Coe, D. M.; O’Connor, S. P.; Tetrahedron Lett. 1995, 36, 2215; Denmark, S. E.; Christenson, B. L.; O'Connor, S. P.; Tetrahedron Lett. 1995, 36, 2219.

21. Charette, A. B.; Brochu, C.; J. Am. Chem. Soc. 1995, 117, 11367. 
22. Charette, A. B.; Juteau, H.; J. Am. Chem. Soc. 1994, 116, 2651; Charette, A. B.; Prescott, S.; Brochu, C.; J. Org. Chem. 1995, 60, 1081.

23. Imai, N.; Takahashi, H.; Kobayashi, S.; Chem. Lett. 1994, 177.

24. Kitajima, H.; Ito, K.; Aoki, Y.; Katsuki, T.; Bull. Chem. Soc. Jpn. 1997, 70, 207.

25. Charette, A. B.; Marcoux, J. -F.; Synlett 1995, 1197.

26. Ukaji, Y.; Nishimura, M.; Fujisawa, T.; Chem. Lett. 1992, 61.

27. Long, J.; Du, H.; Li, K.; Shi, Y.; Tetrahedron Lett. 2005, 46, 2737; Du, H.; Long, J.; Shi, Y.; Org. Lett. 2006, 8, 2827.

28. Davies, H. M. L.; Antoulinakis, E.; Org. React. 2001, 57, 1; Rovis, T.; Evans, D. A.; Prog. Inorg. Chem. 2001, 50, 1; Nishiyama, H.; Enantiomer 1999, 4, 569; Doyle, M. P.; Forbes, D. C.; Chem. Rev. 1998, 98, 911; Singh, V. K.; DattaGupta, A.; Sekar, G.; Synthesis 1997, 137; Ferreira, V. F.; Pereira, L. O. R.; de Souza, M. C. B. V.; Quim. Nova 2001, 24, 540.

29. Putala, M.; Lemenovskii, D. A.; Russ. Chem. Rev. 1994, 63, 197; Li, Y.; Huang, J.-S.; Zhou, Z.-Y.; Che, C.-M.; J. Am. Chem. Soc. 2001, 123, 4843.

30. Pfaltz, A.; Acc. Chem. Res. 1993, 26, 339.

31. Charette, A. B.; Janes, M. K.; Lebel, H.; Tetrahedron: Asymmetry 2003, $14,867$.

32. Nozaki, H.; Moriuti, S.; Takaya, H.; Noyori, R.; Tetrahedron Lett. 1966, 7, 5239; Nozaki, H.; Takaya, H.; Moriuti, S.; Noyori, R.; Tetrahedron 1968, 24, 3655 .

33. Para revisões sobre bis(oxazolinas) em catálise assimétrica, ver: McManus, H. A.; Guiry, P. J.; Chem. Rev. 2004, 104, 4151; Oliveira, A. R. M.; Simonelli, F.; Marques, F. A.; Clososki, G. C.; Oliveira, M. A. F. C.; Quim. Nova 1999, 22, 854

34. Evans, D. A.; Woerpel, K. A.; Hinman, M. M.; Faul, M. M.; J. Am. Chem. Soc. 1991, 113, 726; Evans, D. A.; Woerpel, K. A.; Scott, M. J.; Angew. Chem., Int. Ed. 1992, 31, 430; Davies, D. L.; Kandola, S. K.; Patel, R. K.; Tetrahedron: Asymmetry 2004, 15, 77; Lowental, R. E.; Abiko, A.; Masamune, S.; Tetrahedron Lett. 1990, 31, 6005; Bedekar, A. V.; Andersson, P.; Tetrahedron Lett. 1996, 37, 4073; Müller, D.; Umbricht, G.; Weber, B.; Pfaltz, A.; Helv. Chim. Acta 1991, 74, 232; Harm, A. M.; Knight, J. G.; Stemp, G.; Tetrahedron Lett. 1996, 37, 6189; Uozumi, Y.; Kyota, H.; Kishi, E.; Kitayama, K.; Hayashi, T.; Tetrahedron: Asymmetry 1996, 7, 1603; Imai, Y.; Zhang, W. B.; Kida, T.; Nakatsuji, Y.; Ikeda, I.; J. Org. Chem. 2000, 65, 3326; Knight, J. G.; Belcher, P. E.; Tetrahedron: Asymmetry 2005, 16, 1415; Glos, M.; Reiser, O.; Org. Lett. 2000, 2, 2045; McManus, H. A.; Guiry, P. J.; Chem. Rev. 2004, 104, 4151; Fraile, J. M.; García, J. I.; Herrerías, C. I.; Mayoral, J. A.; Reiser, O.; Vaultier, M.; Tetrahedron Lett. 2004, 45, 6765; Mazet, C.; Köhler, V.; Pfaltz, A.; Angew. Chem., Int. Ed. 2005, 44, 4888; Alexander, K.; Cook, S.; Gibson, C. L.; Tetrahedron Lett. 2000, 41, 7135; Annunziata, R.; Benaglia, M.; Cinquini, M.; Cozzi, F.; Pozzi, G.; Eur. J. Org. Chem. 2003, 1191.

35. Para exemplos recentes de bis(oxazolinas) em ciclopropanações, ver: Itagaki, M.; Masumoto, K.; Yamamoto, Y.; J. Org. Chem. 2005, 70, 3292; Schinnerl, M.; Böhm, C.; Seitz, M.; Reiser, O.; Tetrahedron: Asymmetry 2003, 14, 765; Cornejo, A.; Fraile, J. M.; García, J. I.; Gil, M. J.; Herrerías, C. I.; Legarreta, G.; Martínez-Merino, V.; Mayoral, J. A.; J. Mol. Catal. A: Chem. 2003, 196, 101; Díez-Barra, E.; Fraile, J. M.; García, J. I.; GarcíaVerdugo, E.; Herrerías, C. I.; Luis, S. V.; Mayoral, J. A.; Sánchez-Verdú, P.; Tolosa, J.; Tetrahedron: Asymmetry 2003, 14, 773.

36. Schumacher, R.; Dammast, F.; Reissig, H. -U.; Chem. Eur. J. 1997, 3, 614; Ebinger, A.; Heinz, T.; Umbricht, G.; Pfaltz, A.; Tetrahedron 1998, 54, 10469; Böhm, C.; Schinneri, M.; Bubert, C.; Zabel, M.; Labahn, T.; Parisini, E.; Reiser, O.; Eur. J. Org. Chem. 2000, 2955; Böhm, C.; Reiser, O.; Org. Lett. 2001, 3, 1315; Østergaard, N.; Jensen, J. F.; Tanner, D.; Tetrahedron 2001, 57, 6083.

37. Meng, Q.; Li, M.; Tang, D.; Shen, W.; Zhang, J.; J. Mol. Struct. (Teochem.) 2004, 711, 193.

38. Fraile, J. M.; García, J. I.; Martínez-Merino, V.; Mayoral, J. A.; Salvatella, I.; J. Am. Chem. Soc. 2001, 123, 7616.

39. Honma, M.; Nakada, M.; Tetrahedron Lett. 2003, 44, 9007.

40. Tokunoh, R.; Tomiyama, H.; Sodeoka, M.; Shibasaki, M.; Tetrahedron Lett. 1996, 37, 2449; Sato, H.; Kim, Y. S.; Shibasaki, M.; Tetrahedron Lett. 1999, 40, 2973.

41. Fritschi, H.; Leutenegger, U.; Pfaltz, A.; Angew. Chem., Int. Ed. 1986, 25, 1005; Fritschi, H.; Leutenegger, U.; Siegmann, K.; Pfaltz, A.; Keller, W.; Kratky, C.; Helv. Chim. Acta 1988, 71, 1541; Fritschi, H.; Leutenegger, U.; Pfaltz, A.; Helv. Chim. Acta 1988, 71, 1553; Leutenegger, U.; Umbricht, G.; Fahrni, C.; von Matt, P.; Pfaltz, A.; Tetrahedron 1992, 48, 2143.

42. Lyle, M. P. A.; Wilson, P. D.; Org. Lett. 2004, 6, 855; Ito, K.; Katsuki, T.; Tetrahedron Lett. 1993, 34, 2661; Ito, K.; Katsuki, T.; Synlett 1993, 638; Kwong, H.-L.; Lee, W.-S.; Ng, H.-F.; Chiu, W.-H.; Wong, W.-T.; J. Chem. Soc., Dalton Trans. 1998, 21, 1043.

43. Bayardon, J.; Sinou, D.; Holczknecht, O.; Mercs, L.; Pozzi, G.; Tetrahedron: Asymmetry 2005, 16, 2319.
44. Kanemasa, S.; Hamura, S.; Harada, E.; Yamamoto, H.; Tetrahedron Lett. 1994, 35, 7985

45. Zhang, X.- X.; Fuhrmann, P.; Lippard, S. J.; J. Am. Chem. Soc. 1998, 120, 10260; Rios, R.; Liang, J.; Lo, M. M. C.; Fu, G. C.; Chem. Commun. 2000, 377.

46. Nishiyama, H.; Park, S. B.; Haga, M.; Aoki, K.; Itoh, K.; Chem. Lett. 1994, 1111; Nishiyama, H.; Itoh, Y.; Matsumoto, H.; Park, S.-B.; Itoh, K.; J. Am. Chem. Soc. 1994, 116, 2223; Nishiyama, H.; Itoh, K.; Sugawara, Y.; Matsumoto, H.; Aoki, K.; Itoh, K.; Bull. Chem. Soc. Jpn. 1995, 68, 1247; Nishiyama, H.; Enantiomer 1999, 4, 569; Park, S. -B.; Murata, K.; Matsumoto, H.; Tetrahedron: Asymmetry 1995, 6, 248; Cornejo, A.; Fraile, J. M.; García, J. I.; Gil, M. J.; Luis, S. V.; Martínez-Merino, V.; Mayoral, J. A.; J. Org. Chem. 2005, 70, 5536.

47. Nishiyama, H.; Soeda, N.; Naito, T.; Motoyama, Y.; Tetrahedron: Asymmetry 1998, 9, 2865.

48. Gao, M. Z.; Kong, D.; Clearfield, A.; Zingaro, R. A.; Tetrahedron Lett. 2004, 45, 5649 .

49. Le Maux, P.; Abrunhosa, I.; Berchel, M.; Simonneaux, G.; Gulea, M.; Masson, S.; Tetrahedron: Asymmetry 2004, 15, 2569.

50. Iwasa, S.; Tsushima, S.; Nishiyama, K.; Tsuchiya, Y.; Takesawa, F.; Nishiyama, H.; Tetrahedron: Asymmetry 2003, 14, 855.

51. Simpson, J. H.; Godfrey, J.; Fox, R.; Kotnis, A.; Kacsur, D.; Hamm, J.; Totelben, M.; Rosso, V.; Mueller, R.; Delaney, E.; Deshpande, R. P.; Tetrahedron: Asymmetry 2003, 14, 3569 .

52. Marcin, L. R.; Denhart, D. J.; Mattson, R. J.; Org. Lett. 2005, 7, 2651.

53. Simonneaux, G.; Le Maux, P.; Coord. Chem. Rev. 2002, 228, 43; PaulRoth, C.; De Montigny, F.; Rethoré, G.; Simonneaux, G.; Gulea, M.; Masson, S.; J. Mol. Catal. A: Chem. 2003, 201, 79; Paul-Roth, C.; RaultBerthelot, J.; Simonneaux, G.; Tetrahedron 2004, 60, 12169; Ferrand, Y.; Le Maux, P.; Simonneaux, G.; Tetrahedron: Asymmetry 2005, 16, 3829.

54. Lo, W. -C.; Che, C. -M.; Cheng, K. -F.; Mak, T. C. W.; J. Chem. Soc., Chem. Commun. 1997, 1205; Zhang, J. -L.; Che, C. -M.; Org. Lett. 2002, 4, 1911 ; Ferrand, Y.; Poriel, C.; Le Maux, P.; Rault-Berthelot, J.; Simonneaux, G.; Tetrahedron: Asymmetry 2005, 16, 1463.

55. Munslow, I. J.; Gillespie, K. M.; Deeth, R. J.; Scott, P.; J. Chem. Soc., Chem. Commun. 2001, 1638; Tang, W.; Hu, X.; Zhang, X.; Tetrahedron Lett. 2002, $43,3075$.

56. Zhang, Z.; Yao, X.; Li, C.; Chen, H.; Hu, X.; Tetrahedron Lett. 2001, 43, 2847; Uchida, T.; Irie, R.; Katsuki, T.; Tetrahedron 2000, 56, 3501.

57. Bachmann, S.; Furler, M.; Mezzetti, A.; Organometallics 2001, 20, 2102; Bachmann, S.; Mezzetti, A.; Helv. Chim. Acta 2001, 84, 3063.

58. Bonaccorsi, C.; Bachmann, S.; Mezzetti, A.; Tetrahedron: Asymmetry 2003, 14,845 .

59. Miller, J. A.; Jin, W. C.; Nguyen, S. T.; Angew. Chem., Int. Ed. 2002, 41, 2953.

60. Ikeno, T.; Sato, M.; Yamada, T.; Chem. Lett. 1999, 1345; Yamada, T.; Ikeno, T.; Sekino, H.; Sato, M.; Chem. Lett. 1999, 719; Ikeno, T.; Nishizuka, A.; Sato, M.; Yamada, T.; Synlett 2001, 406; Ikeno, T.; Sato, M.; Sekino, H.; Nishizuka, A.; Yamada, T.; Bull. Chem. Soc. Jpn. 2001, 74, 2139; Ikeno, T.; Iwakura, I.; Yamada, T.; Bull. Chem. Soc. Jpn. 2001, 74, 2151; Ikeno, T.; Iwakura, I.; Yabushita, S.; Yamada, T.; Org. Lett. 2002, 4, 517.

61. Fukuda, T.; Katsuki, T.; Synlett 1995, 825; Fukuda, T.; Katsuki, T.; Tetrahedron 1997, 53, 7201.

62. Ito, Y. N.; Katsuki, T.; Bull. Chem. Soc. Jpn. 1999, 72, 603; Niimi, T.; Uchida, T.; Irie, R.; Katsuki, T.; Tetrahedron Lett. 2000, 41, 3647; Niimi, T.; Uchida, T.; Irie, R.; Katsuki, T.; Adv. Synth. Catal. 2001, 343, 79; Uchida, T.; Saha, B.; Batsuki, T.; Tetrahedron Lett. 2001, 42, 2521.

63. Saba, B.; Uchida, T.; Katsuki, T.; Tetrahedron: Asymmetry 2003, 14, 823. 64. Chen, Y.; Zhang, P.; J. Org. Chem. 2004, 69, 2431.

65. Chen, Y.; Zhang, X. P.; Synthesis 2006, 1697.

66. Chen, Y.; Gao, G. -Y.; Zhang, X. P.; Tetrahedron Lett. 2005, 46, 4965.

67. Ishitani, H.; Achiwa, K.; Synlett 1997, 781.

68. Hashimoto, S.; Watanabe, N.; Ikegami, S.; Tetrahedron Lett. 1990, 31, 5173; Watanabe, N.; Ogawa, T.; Ohtake, Y.; Ikegami, S.; Hashimoto, S.; Synlett 1996, 85.

69. Estevan, F.; Lahuerta, P.; Lloret, J.; Penno, D.; Sanaú, M.; Úbeda, M. A.; J. Organomet. Chem. 2005, 690, 4424.

70. Doyle, M. P.; Winchester, W. R.; Hoorn, J. A. A.; Lynch, V.; Simonsen, S. H.; Ghosh, R.; J. Am. Chem. Soc. 1993, 115, 9968.

71. Doyle, M. P.; Zhou, Q. L.; Simonsen, S. H.; Lynch, V.; Synlett 1996, 697; Müller, P.; Grass, S.; Shahi, S. P.; Bernardinelli, G.; Tetrahedron 2004, 60, 4755; Hu, W.; Timmons, D. J.; Doyle, M. P.; Org. Lett. 2002, 4, 901.

72. Teng, P. -F.; Lai. T. -S.; Kwong, H. -L.; Che, C. -M.; Tetrahedron: Asymmetry 2003, 14, 837.

73. Doyle, M. P.; Davies, S. B.; Hu, W.; Org. Lett. 2000, 2, 1145

74. Müller, P.; Allenbach, Y.; Robert, E.; Tetrahedron: Asymmetry 2003, 14, 779; Müller, P.; Acc. Chem. Res. 2004, 37, 243. 
75. Shi, W.; Zhang, B.; Zhang, J.; Liu, B.; Zhang, S.; Wang, J.; Org. Lett. 2005 7, 3103; Nakamura, E.; Yoshikai, N.; Yamanaka, M.; J. Am. Chem. Soc. 2002, 124, 7181; Müller, P.; Allenbach, F. Y.; Chappellet, S.; Ghanem, A.; Synthesis 2006, 1689; Müller, P.; Bernardinelli, G.; Allenbach, Y. F.; Ferri, M.; Flack, H. D.; Org. Lett. 2004, 6, 1725; Davies, H. M. L.; Panaro, S. A.; Tetrahedron 2000, 56, 4871; Davies, H. M. L.; Loe, Ø.; Synthesis 2004, 16, 2595.

76. Para uma descrição das abordagens em síntese assimétrica, ver: Pinheiro, S.; Ferreira, V. F.; Quim. Nova 1998, 21, 312.

77. Para uma revisão sobre ciclopropanação de carboidratos, ver: Yu, M.; Pagenkopf, B. L.; Tetrahedron 2005, 61, 321.

78. Furukawa, J.; Kawabata, N.; Nishimura, J.; Tetrahedron Lett. 1966, 7, 3353 Lorenz, J. C.; Long, J.; Yang, Z.; Xue, S.; Xie, Y.; Shi, Y.; J. Org. Chem. 2004, 69, 327.

79. Hoberg, J. O.; Claffey, D. J.; Tetrahedron Lett. 1996, 37, 2533; Timmers, C. M.; Leeuwenburgh, M. A.; Verheijen, J. C.; Vandermarel, G. A.; van Boom, J. H.; Tetrahedron: Asymmetry 1996, 7, 49; Schumacher, R.; Reissig, H. -U.; Synlett 1996, 1121.
80. Dötz, K. H.; Jäkel, C.; Haase, W. -C.; J. Organomet. Chem. 2001, 617, 119.

81. Ferreira, M. L. G.; Pinheiro, S.; Perrone, C. C.; Costa, P. R. R.; Ferreira, V. F.; Tetrahedron: Asymmetry 1998, 9, 2671; Ferreira, V. F.; Pinheiro, S.; Perrone, C. C.; Costa, P. R. R.; J. Braz. Chem. Soc. 2000, 11, 266.

82. Costa, P. R. R.; Ferreira, V. F.; Alencar, K. G.; Araújo Filho, H. C.; Ferreira, C. M.; Pinheiro, S.; J. Carbohydr. Chem. 1996, 15, 69; Costa, P. R. R.; Ferreira, V. F.; Araújo Filho, H. C.; Pinheiro, S.; J. Braz. Chem. Soc. 1996, 7, 67.

83. Pinheiro, S.; Pedraza, S. F.; Peralta, M. A.; Carvalho, E. M.; Farias, F. M. C.; Ferreira, V. F.; J. Carbohydr. Chem. 1998, 17, 901.

84. Ribeiro, C. M. R.; Santos, E. S.; Jardim, A. H. O.; Maia, M. P.; da Silva, F. C.; Moreira, A. P. D.; Ferreira, V. F.; Tetrahedron: Asymmetry 2002, 13, 1703.

85. Diéguez, M.; Pàmies, O.; Ruiz, A.; Díaz, Y.; Castillón, S.; Claver, C.; Coord. Chem. Rev. 2004, 248, 2165; Borriello, C.; Cucciolito, M. E.; Panunzi, A.; Rufo, F.; Tetrahedron: Asymmetry 2001, 12, 2467.

86. Holland, D. A.; Laidler, D. J.; Milner, X.; J. Mol. Catal. 1981, 11, 119 